\title{
MULTI-SCALE METHODS FOR WAVE PROPAGATION IN HETEROGENEOUS MEDIA*
}

\author{
BJÖRN ENGQUIST ${ }^{\dagger}$, HENRIK HOLST ${ }^{\ddagger}$, AND OLOF RUNBORG ${ }^{\S}$
}

\begin{abstract}
Multi-scale wave propagation problems are computationally costly to solve by traditional techniques because the smallest scales must be represented over a domain determined by the largest scales of the problem. We have developed and analyzed new numerical methods for multi-scale wave propagation in the framework of heterogeneous multi-scale method. The numerical methods couple simulations on macro- and micro-scales for problems with rapidly oscillating coefficients. We show that the complexity of the new method is significantly lower than that of traditional techniques with a computational cost that is essentially independent of the micro-scale. A convergence proof is given and numerical results are presented for periodic problems in one, two, and three dimensions. The method is also successfully applied to non-periodic problems and for long time integration where dispersive effects occur.
\end{abstract}

Key words. Multi-scale, wave propagation, HMM, homogenization.

AMS subject classifications. 65N06, 35B27, 35L05.

\section{Introduction}

We consider the initial boundary value problem for the scalar wave equation,

$$
\begin{cases}u_{t t}^{\varepsilon}-\nabla \cdot A^{\varepsilon} \nabla u^{\varepsilon}=0, & \Omega \times\{0 \leq t \leq T\}, \\ u^{\varepsilon}=f, \quad u_{t}^{\varepsilon}=g, & \Omega \times\{t=0\},\end{cases}
$$

on a smooth domain $\Omega \subset \mathbb{R}^{N}$ with $A^{\varepsilon}(x)$ a symmetric, uniformly positive definite matrix. The expression $\nabla \cdot A^{\varepsilon} \nabla u^{\varepsilon}$ should be interpreted as $\nabla \cdot\left(A^{\varepsilon} \nabla u^{\varepsilon}\right)$. We assume that $A^{\varepsilon}$ has oscillations on a scale proportional to $\varepsilon \ll 1$. The solution of (1.1) will then also be highly oscillating in both time and spatial directions on the scale $\varepsilon$. It is typically very computationally costly to solve these kinds of multi-scale problems by traditional numerical techniques. The smallest scale must be well represented over a domain, which is determined by the largest scales. For wave propagation small scales may also originate from high frequencies in initial data or boundary data. We will however focus on the case when they come from strong variations in the wave velocity field. Such variable velocity problems occur for example in seismic wave propagation in subsurface domains with inhomogeneous material properties and microwave propagation in complex geometries.

Recently, new frameworks for numerical multi-scale methods have been proposed, including the heterogeneous multi-scale method (HMM) [5] and the equation free methods [13]. These methods couple simulations on macro- and micro-scales. We use HMM $[5,6,4]$, in which a numerical macro-scale method gets necessary information

*Received: November 12, 2009; accepted (in revised version): April 19, 2010. Communicated by Shi Jin.

This research was supported by the National Science Foundation grant DMS 0714612 and by the Swedish Foundation for Strategic Research through the programme "Advanced Multiscale Techniques for New Discoveries in Science and Engineering", Doss No A3 02:124.

${ }^{\dagger}$ Department of Mathematics and Institute for Computational Engineering and Sciences, The University of Texas at Austin, 1 University Station C1200, Austin TX 78712, U.S.A. (engquist@ices.utexas.edu).

${ }^{\ddagger}$ Department of Numerical Analysis, CSC, KTH, 10044 Stockholm, Sweden (holst@nada.kth.se).

$\S$ Department of Numerical Analysis, CSC, KTH, 10044 Stockholm, Sweden (olofr@nada.kth.se), and Swedish e-Science Research Center (SeRC). 
from micro-scale models that are only solved on small sub domains. This framework has been applied to a number multi-scale problems, for example, ODEs with multiple time scales [10], elliptic and parabolic equations with multi-scale coefficients $[7,17,1]$, kinetic schemes [6], and large scale MD simulation of gas dynamics [15].

On the macro-scale we will assume a simple flux form,

$$
\tilde{u}_{t t}-\nabla \cdot F=0,
$$

in our HMM approximation of the wave equation (1.1). The solution $\tilde{u}$ should be a good approximation of the solution to (1.1) and the value of $F$ on the macro-scale grid is computed by numerically approximating (1.1) on small micro-scale domains.

The goal of our research is to better understand the HMM process with wave propagation as an example and also to derive computational techniques for future practical wave equation applications. One contribution is a convergence proof in the multidimensional case that includes a discussion on computational complexity. The analysis is partially based on the mathematical homogenization theory for coefficients $A^{\varepsilon}$ with periodic oscillations $[2,3]$.

Classical homogenization considers partial differential equations with rapidly oscillating coefficients. As the period of the coefficients in the PDE goes to zero, the solution approaches the solution to another PDE, a homogenized PDE. The coefficients in the homogenized PDE have no $\varepsilon$ dependency. For example, in the setting of composite materials consisting of two or more mixed constituents (i.e., thin $\varepsilon$ periodic laminated layers), homogenization theory gives the macroscopic properties of the composite. It is interesting to note that the macroscopic properties are often different than the average of the individual constituents that makes up the composite [3]. The wave equation (1.1), with $A^{\varepsilon}(x)=A(x, x / \varepsilon)$ and $A(x, y)$ periodic in $y$, has an homogenized equation,

$$
\begin{cases}\bar{u}_{t t}-\nabla \cdot \bar{A} \nabla \bar{u}=0, & \Omega \times\{0 \leq t \leq T\}, \\ \bar{u}=\bar{f}, \quad \bar{u}_{t}=\bar{g}, \quad \Omega \times\{t=0\},\end{cases}
$$

where $\bar{A}(x)$ is called the homogenized or effective coefficient. The homogenized solution $\bar{u}$ can be used as an approximation of the solution $u^{\varepsilon}$ of the full equation since $u^{\varepsilon}(x)=\bar{u}(x)+\mathcal{O}(\varepsilon)$. Note that the homogenized equations are often less expensive to solve with numerical methods, since the coefficients varies slowly without $\varepsilon$ variations. We refer to $[2,18,3,12,16,9]$ for more about homogenization in general.

It should be noted that even if our numerical methods use ideas from homogenization theory they do not solve the homogenized equations directly. The goal is to develop computational techniques that can be used when there is no known homogenized equation available. In the research presented here many of the homogenized equations are actually available and could in practice be numerically directly approximated. We have chosen this case in order to be able to develop a rigorous convergence analysis and to have a well-understood environment for numerical tests. We also apply the techniques to problems that does not fit the theory. In example 4.2 .3 an equation with non-periodic coefficients is approximated and in example 4.5 an equation is solved over very long time. The latter is particularly interesting since the homogenized solution contains dispersive effects, which influence the solution for $t=\mathcal{O}\left(\varepsilon^{-2}\right)$. This dispersive process is captured by a high accuracy HMM technique without explicit approximation of any dispersive term.

The article is organized as follows. In section 2 we first discuss the HMM framework in a general setting and thereafter in section 2.1 our HMM method for the wave 
equation. We give a rigorous proof of the approximation error by the HMM method in the periodic coefficient case in section 3 . In section 4 we show numerical results, which also includes a non-periodic problem and an example with very long time. The last section 5 ends this paper with our conclusions.

\section{Heterogeneous multi-scale methods (HMM)}

In the HMM framework, the general setting of a multi-scale problem is the following: we assume that there exist two models, a micro model $f(u, d)=0$ describing the full problem and a coarse macro model $F(\tilde{u}, \tilde{d})=0$. The micro model is accurate but is expensive to compute by traditional methods. The macro model gives a coarse scale or low frequency solution $\tilde{u}$, which is assumed to be a good approximation of the micro-scale solution $u$ and is less expensive to compute. The model is however incomplete in some sense and requires additional data. We assume that $F(\tilde{u}, \tilde{d})=0$ can still be discretized by a numerical method, called the macro solver. A key idea in the HMM method is to provide the missing data in the macro model $(\tilde{d})$ using a local solution to the micro model. The micro model solution $u$ is computed locally on a small domain with size proportional to the micro-scale. The initial data and boundary conditions $(d)$ for this computation is constrained by the macro-scale solution $\tilde{u}$.

2.1. HMM for the wave equation. We will formulate a general HMM framework for the wave equation on the domain $Y=[0,1]^{d}$. Let $u^{\varepsilon}$ be $Y$-periodic and solve,

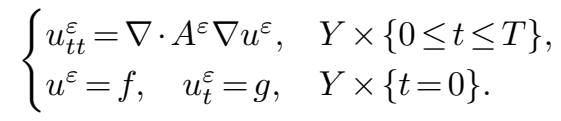

We follow the same strategy as in [1] for parabolic equations and in [19] for the onedimensional advection equation. See also [8]. We assume there exists a macro-scale PDE of the form

$$
\begin{cases}u_{t t}-\nabla \cdot F(x, u, \nabla u, \ldots)=0, & Y \times\{0 \leq t \leq T\}, \\ u=f, \quad u_{t}=g, & Y \times\{t=0\} \\ u, & Y \text {-periodic, }\end{cases}
$$

where $F$ is a function of $x, u$, and higher derivatives of $u$. The assumption on (2.2) is that $u \approx u^{\varepsilon}$ when $\varepsilon$ is small. In the clean homogenization case we would have $F=\bar{A} \nabla u$, but we will not assume knowledge of a homogenized equation. Instead we will solve the PDE (2.1), only in a small time and space box, and from that solution extract a value for $F$. The form of the initial data for this micro problem will be determined from the local behavior of $u$. In the method we suppose that $F=F(x, \nabla u)$.

Step 1: Macro model discretization. We discretize (2.2) using central differences with time step $K$ and spatial grid size $H$ in all directions,

$$
\left\{\begin{array}{l}
U_{m}^{n+1}=2 U_{m}^{n}-U_{m}^{n-1}+\frac{K^{2}}{H}\left(F_{m+\frac{1}{2} e_{1}}^{(1)}-F_{m-\frac{1}{2} e_{1}}^{(1)}\right)+\cdots+\frac{K^{2}}{H}\left(F_{m+\frac{1}{2} e_{d}}^{(d)}-F_{m-\frac{1}{2} e_{d}}^{(d)}\right), \\
F_{m-\frac{1}{2} e_{k}}^{n}=F\left(x_{m-\frac{1}{2} e_{k}}, P_{m-\frac{1}{2} e_{k}}^{n}\right), \quad k=1, \ldots, d, \quad \text { (Note: } F_{m-\frac{1}{2} e_{k}}^{n} \text { is a vector), }
\end{array}\right.
$$

where $F_{m \pm 1 / 2 e_{k}}^{n}$ is $F$ evaluated at point $x_{m \pm 1 / 2 e_{k}}$. The quantity $P_{m \pm \frac{1}{2} e_{k}}^{n}$ approximates $\nabla u$ in the point $x_{m \pm 1 / 2 e_{k}}$. In figure 2.1 we show an example of the numerical scheme in two dimensions. There $P_{m+\frac{1}{2} e_{2}}^{n}$ is given by the expression (A.4) in the Appendix. The macro discretization $H$ and $K$ is supposed to be fine enough to capture all information on the macroscopic scale. 


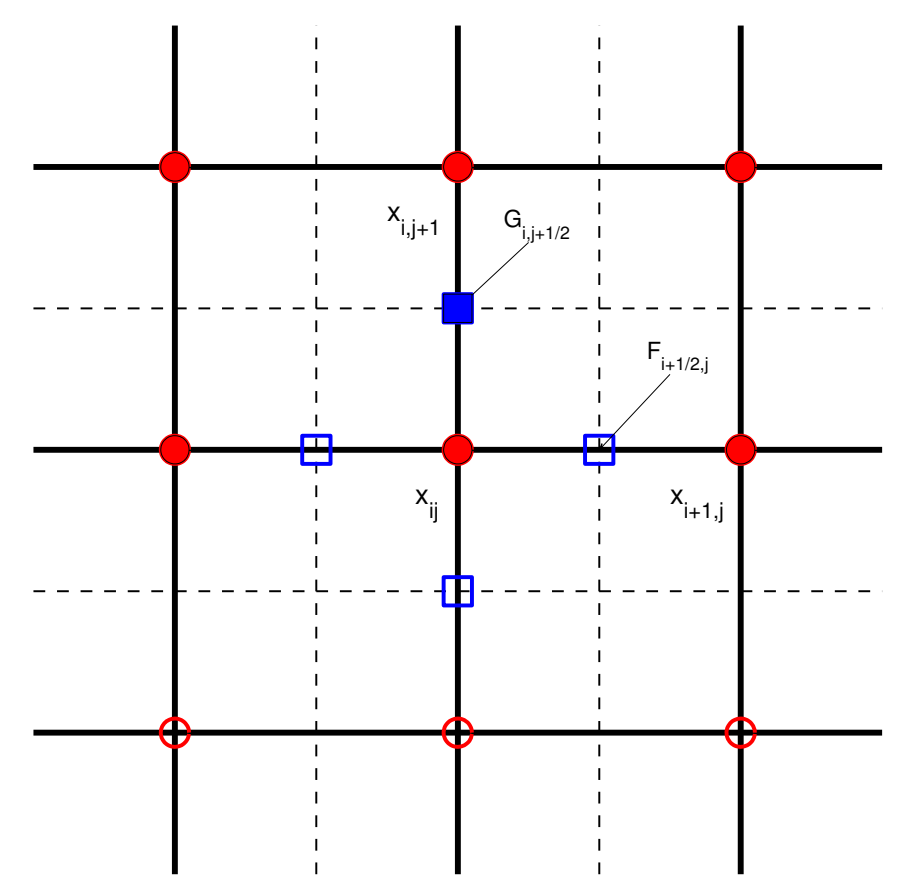

FIG. 2.1. The Numerical scheme (A.4) for $P$ in two dimensions. In the figure above the two components of $F$ in two different positions are given by $F_{i+1 / 2, j}$ and $G_{i, j+1 / 2}$. The $U$ points involved in computing $F_{m+\frac{1}{2} e_{2}}^{n}=G_{i, j+1 / 2}$ and $\nabla u \approx P_{m+\frac{1}{2} e_{2}}^{n}$ are indicated by filled circles. Note that the squares are where either $\left(A_{11}^{\varepsilon} \partial_{x}+A_{12}^{\varepsilon} \partial_{y}\right) u^{\varepsilon}$ or $\left(A_{21}^{\varepsilon} \partial_{x}+A_{22}^{\varepsilon} \partial_{y}\right) u^{\varepsilon}$ are computed, and not the full gradient $A^{\varepsilon} \nabla u^{\varepsilon}$.

Step 2: Micro problem. The evaluation of $F_{m-\frac{1}{2} e_{k}}^{n}$ in each grid point is done by solving a micro problem to fill in the missing data in the macro model. Given the parameters $x_{m-\frac{1}{2} e_{k}}$ and $P_{m-\frac{1}{2} e_{k}}^{n}$, we solve a corresponding micro problem over a small micro box $Y^{\varepsilon}$, centered around $x_{m-\frac{1}{2} e_{k}}$. In order to simplify the notation, we make a change of variables $x-x_{m-\frac{1}{2} e_{k}} \mapsto x$. This implies that $A^{\varepsilon}(x) \mapsto A^{\varepsilon}\left(x+x_{m-\frac{1}{2} e_{k}}\right)$. The micro problem has the form,

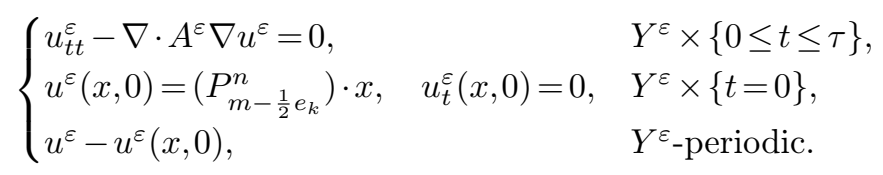

We see that there is a strong influence from the macro to micro scale due to the initial data. The micro discretization $h$ and $k$ must be such that the solution to (2.4) is resolved for the given initial data.

We keep the micro box size of order $\varepsilon$, i.e. $\tau$, $\operatorname{diam} Y^{\varepsilon}=\mathcal{O}(\varepsilon)$. We note that the solution $u^{\varepsilon}$ is an even function with respect to $t$ (i.e. $\left.u^{\varepsilon}(x,-t)=u^{\varepsilon}(x, t)\right)$ due to the initial condition $u_{t}^{\varepsilon}(x, 0)=0$.

Step 3: Reconstruction step. After we have solved for $u^{\varepsilon}$ for all $Y^{\varepsilon} \times[0, \tau]$ we approximate $F_{m-\frac{1}{2} e_{k}}^{n} \approx \tilde{F}\left(x_{m-\frac{1}{2} e_{k}}, P_{m-\frac{1}{2} e_{k}}^{n}\right)$. The function $\tilde{F}$ is the mean value of $f^{\varepsilon}=A^{\varepsilon} \nabla u^{\varepsilon}$ over $[-\eta, \eta]^{d} \times[-\tau, \tau]$ where $[-\eta, \eta]^{d} \subset Y^{\varepsilon}$. The approximation can be 
improved with respect to the size of $\tau / \varepsilon$ and $\eta / \varepsilon$ by computing a weighted average of $f^{\varepsilon}$. We consider kernels $K$ described in [10]: we let $\mathbb{K}^{p, q}$ denote the kernel space of functions $K$ such that $K \in C_{c}^{q}(\mathbb{R})$ with supp $K=[-1,1]$ and

$$
\int K(t) t^{r} \mathrm{~d} t= \begin{cases}1, & r=0 \\ 0, & 1 \leq r \leq p\end{cases}
$$

Furthermore we will denote $K_{\eta}$ as a scaling of $K$

$$
K_{\eta}(x):=\frac{1}{\eta} K\left(\frac{x}{\eta}\right), \quad K \in \mathbb{K}^{p, q},
$$

with compact support in $[-\eta, \eta]$. We use kernels of this sort to improve the approximation quality for the mean value computation,

$$
\tilde{F}\left(x_{m-\frac{1}{2} e_{k}}, P_{m-\frac{1}{2} e_{k}}^{n}\right)=\iint K_{\tau}(t) K_{\eta}(x) f_{k}^{\varepsilon} \mathrm{d} x \mathrm{~d} t, \quad f^{\varepsilon}=A^{\varepsilon}\left(x+x_{m+\frac{1}{2} e_{k}}\right) \nabla u^{\varepsilon},
$$

where here the multi variable kernel $K_{\eta}(x)$ is defined as

$$
K_{\eta}(x)=K_{\eta}\left(x_{1}\right) K_{\eta}\left(x_{2}\right) \cdots K_{\eta}\left(x_{d}\right),
$$

using the single valued kernel $K_{\eta}$, still denoted by $K_{\eta}$. The domain $Y^{\varepsilon}$ is chosen such that $[-\eta, \eta]^{d} \subset Y^{\varepsilon}$ and sufficiently large for information not to propagate into the region $[-\eta, \eta]^{d}$. Typically we use

$$
Y=\left[-y_{\max }, y_{\max }\right]^{d}, \quad y_{\max }=\eta+\tau \sqrt{\sup \left\|A^{\varepsilon}\right\|_{2}},
$$

c.f. discussion about micro solver boundary conditions in [19]. In this way we do not need to worry about the effects of boundary conditions. Note therefore that other types of boundary conditions could also be used in (2.4).

REMARK 2.1. It is possible to find functions with infinite $q$. In [10] a kernel $K_{\exp }$ is given, where $p=1$ and $q$ is infinite:

$$
K_{\exp }(x)= \begin{cases}C_{0} \exp \left(\frac{5}{x^{2}-1}\right), & |x|<1, \\ 0, & |x| \geq 1,\end{cases}
$$

where $C_{0}$ is chosen such that $\int K_{\exp }(x) \mathrm{d} x=1$. This kernel is suitable for problems where $A^{\varepsilon}$ is of the form $A^{\varepsilon}(x)=A(x / \varepsilon)$.

REMARK 2.2. The weighted integrals above are computed numerically with a simple trapezoidal rule.

REMARK 2.3. In our implementation, the micro problem (2.4) is solved with the same numerical scheme as the macro problem (2.3).

2.2. Computational cost. We will now discuss an alternative to computing (2.5) on-the-fly in the macro solver. In some cases, when the dimension and the number of parameters in the problem are few, a sequential coupling can prove to be more efficient. We stress that the algorithm suggested here does not require precomputing and all analysis are made for on-the-fly computations. 
Let us assume that the time step is proportional to $\varepsilon$ in all direct solvers. Using a direct solver for $(2.1)$ on the full domain implies a cost of order $\varepsilon^{-(d+1)}$. The total cost for on-the-fly HMM is of the form (cost of micro problem) $\times M_{d}$ where

$$
M_{d} \sim \frac{1}{K} \cdot \frac{1}{H^{d}}
$$

is the number of micro problems needed to be solved. The cost of a single micro problem is of the form $(\tau / \varepsilon) \times(\eta / \varepsilon)^{d}$. We assume kernels with $\tau, \eta \sim \varepsilon$ and that $M_{d}$ does not depend on $\varepsilon$. With these assumption our HMM method has a computational cost independent of $\varepsilon$. The constant can, however, still be large as it is proportional to $M_{d}$ which depends inversely on $K$ and $H^{d}$. Fortunately the computational cost of the HMM process can be reduced significantly. We observe that the function $\tilde{F}(x, p)$ in (2.5) is linear in $p$. It is in fact composed of three linear operations:

1. Compute initial data $u(x, 0)$ and $u_{t}(x, 0)$ from $p, u(x, 0)=p \cdot x$.

2. Solve $u_{t t}^{\varepsilon}-\nabla \cdot A^{\varepsilon} \nabla u^{\varepsilon}=0$ for $0 \leq t \leq \tau$.

3. Compute average $\tilde{F}=\iint K_{\tau} K_{\eta} f^{\varepsilon} \mathrm{d} x \mathrm{~d} t$ where $f^{\varepsilon}=A^{\varepsilon} \nabla u^{\varepsilon}$

The first operation is clearly a linear operation. In step two we compute a solution to a linear PDE, therefore this step is linear as well. Computing the integral average in step three is also a linear operation.

As a corollary we can apply the HMM process to a smaller number of micro problems and form linear combinations of those for any given $\tilde{F}$ computation. More precisely, after precomputing $\tilde{F}\left(x, e_{i}\right), i=1,2, \ldots, d$ we can compute $\tilde{F}$ for fixed $x \in \Omega$ and any $p \in \mathbb{R}^{d}$,

$$
\tilde{F}(x, p)=\sum_{i=1}^{d} p_{i} \tilde{F}\left(x, e_{i}\right),
$$

where $p_{i}$ is the $i$ th coefficient in $p$ in the basis $e_{1}, e_{2}, \ldots, e_{d}$. In conclusion, by precomputing the micro problems $\tilde{F}\left(x_{m}, e_{i}\right)$ in (2.9) we only need to solve $d$ micro problems in each macro grid point $x_{m}=m H$. There is no need to solve any micro problems again in the next macro time step. The complexity is as before $\mathcal{O}(1)$ in $\varepsilon$, but now $M_{d} \sim d / H^{d}$ which means a smaller constant not depending on the number of time steps. Note that this works if $A^{\varepsilon}$ does not depend on $t$. If $A^{\varepsilon}$ depends on $t$ or is nonlinear in $u$ it would in general not be as cost effective to precompute $\tilde{F}$.

REMARK 2.4. In fact, if $A^{\varepsilon}$ is $\varepsilon$-periodic and the macro grid is such that $x_{m}=r$ ( $\bmod \varepsilon$ ), where $r$ is constant and independent of $m$, we only need to solve $d$ micro problems in total, i.e., $M_{d}=d$. In this case, the total cost is independent of both $\varepsilon$ and the macro grid sizes $H, K$.

REMARK 2.5. The macro scheme suggested here is embarrassingly parallel in space. This fact has been exploited by the authors in a Message Passing Interface (MPI) code. We think that it would be possible to implement the same algorithm in a general purpose GPU environment and see a good utilization of the hardware.

\section{Convergence theory}

In this section we apply the HMM process to the problem (1.1) with $A^{\varepsilon}(x)=$ $A(x / \varepsilon)$ where $A$ is a $Y$-periodic symmetric positive matrix and show that it generates results close to a direct discretization of the homogenized equation (1.3). We will 
show a convergence result for smooth (i.e., $C^{\infty}$ ) solutions and data. In particular we show that

$$
\tilde{F}(x, p)=F(x, p)+\mathcal{O}\left(\left(\frac{\varepsilon}{\eta}\right)^{q}\right) .
$$

The functions $\tilde{F}$ and $F$ are defined in (2.5) and (2.2) respectively and we note that here $F(x, p)=\bar{A} p$ where $\bar{A}$ is the homogenized coefficient. The integer $q$ depends on the smoothness of the kernel used to compute the weighted average of $f^{\varepsilon}$ in (2.5).

We will formulate the problem in the setting of elliptic operators. For the analysis we solve the micro problem (2.4) over all of $\mathbb{R}^{d}$

$$
\begin{cases}u_{t t}^{\varepsilon}-\nabla \cdot A^{\varepsilon} \nabla u^{\varepsilon}=0, & \mathbb{R}^{d} \times\{0 \leq t \leq \tau\}, \\ u^{\varepsilon}=p \cdot x, \quad u_{t}^{\varepsilon}=0, & \mathbb{R}^{d} \times\{t=0\} .\end{cases}
$$

Note that this gives the same $\tilde{F}$ as in (2.5) if we choose a sufficiently large box $Y^{\varepsilon}$.

THEOREM 3.1. Let $\tilde{F}\left(x_{0}, p\right)$ be defined by (2.5), where $u^{\varepsilon}$ solves the micro problem (3.2) exactly, $A^{\varepsilon}(x)=A(x / \varepsilon)$, and $A$ is $Y$-periodic and $C^{\infty}$. Moreover suppose $K \in$ $\mathbb{K}^{p^{\prime}, q}, f$ and $g$ are $C^{\infty}$ and $\tau=\eta$. Then for $p \neq 0$,

$$
\frac{1}{p}\left|\tilde{F}\left(x_{0}, p\right)-F\left(x_{0}, p\right)\right| \leq C\left(\frac{\varepsilon}{\eta}\right)^{q}
$$

where $C$ is independent of $\varepsilon, \eta, p$ and $q$. Furthermore, for the numerical approximation given in (2.3) in one dimension, with $H=n \varepsilon$ for some integer $n$ and smooth initial data, we have the error estimate:

$$
\left|U_{m}^{n}-\bar{u}\left(x_{m}, t_{n}\right)\right| \leq C(T)\left(H^{2}+(\varepsilon / \eta)^{q}\right), \quad 0 \leq t_{n} \leq T,
$$

where $\bar{u}$ is the homogenized solution to (1.3).

Proof. We will prove the theorem in the following steps:

1. Reformulate the problem as a PDE for a periodic function.

2. Define an elliptic operator $L(y)$.

3. Expand $\nabla_{y} \cdot A(y)$ and $v(y, t)$ (to be defined) in eigenfunctions of $L(y)$.

4. Compute time dependent $v_{j}(t)$ coefficients in the above eigenfunction expansion.

5. Compute the integral of $f^{\varepsilon}$ to get $\hat{F}$.

6. Compute the solution to a cell problem and give final estimate.

Step 1. Express the solution to (3.2) as

$$
u^{\varepsilon}(t, x)=p \cdot x+v(x / \varepsilon, t) .
$$

We insert this into (3.2) to get a PDE for $v$

$$
\left\{\begin{array}{l}
v_{t t}=\frac{1}{\varepsilon} \nabla_{y} \cdot A(y) p+\frac{1}{\varepsilon^{2}} \nabla_{y} \cdot A(y) \nabla_{y} v(y) \\
v(x, 0)=0, \quad v_{t}(x, 0)=0
\end{array}\right.
$$

where $y=x / \varepsilon$. Since $A$ is $Y$-periodic, so is $v$, and we can solve (3.4) as a $Y$-periodic problem. 
Step 2. We define the linear operator $L(y):=-\nabla_{y} \cdot A(y) \nabla_{y}$ on $Y$ with periodic boundary conditions. Denote $w_{j}(y)$ to be the eigenfunctions and $\lambda_{j}$ to be the corresponding (non-negative) eigenvalues of $L$. Since $L$ is uniformly elliptic, standard theory on periodic elliptic operators informs us that all eigenvalues are strictly positive, bounded away from zero, except for the single zero eigenvalue [14]

$$
0=\lambda_{0}<\lambda_{1} \leq \lambda_{2} \leq \ldots
$$

and $w_{j} \in C^{\infty}$ forms an orthonormal basis for $L_{\mathrm{per}}^{2}(Y)$. Also note that $w_{0}=|Y|^{-1}$ is a constant function.

Step 3. We express $\nabla_{y} \cdot A(y)$ and $v(y)$ in eigenfunctions of $L$ :

$$
\nabla_{y} \cdot A(y)=\sum_{j=1}^{\infty} a_{j} w_{j}(y) \quad \text { and } \quad v(y, t)=\sum_{j=0}^{\infty} v_{j}(t) w_{j}(y)
$$

Note that here $a_{j}$ are column vectors and as in the one dimensional case we have that $a_{0}=0$ since the mean value of $\nabla_{y} \cdot A(y)$ is zero,

$$
a_{0}=\int_{Y} \nabla_{y} \cdot A(y) w_{0}(y) \mathrm{d} y=\frac{1}{|Y|} \int_{Y} \nabla_{y} \cdot A(y) \mathrm{d} y=0
$$

Step 4. We plug the eigenfunction expansions (3.6) into (3.4) and find that

$$
\sum_{j=0}^{\infty} v_{j}^{\prime \prime} w_{j}=\frac{1}{\varepsilon} p \cdot \sum_{j=1}^{\infty} a_{j} w_{j}-\frac{1}{\varepsilon^{2}} \sum_{j=1}^{\infty} L v_{j} w_{j}=\sum_{j=1}^{\infty} \frac{p \cdot a_{j}}{\varepsilon} w_{j}-\sum_{j=1}^{\infty} \frac{\lambda_{j}}{\varepsilon^{2}} v_{j} w_{j} .
$$

By collecting terms of $w_{j}$ we obtain

$$
v_{j}^{\prime \prime}+\frac{\lambda_{j}}{\varepsilon^{2}} v_{j}=\frac{p \cdot a_{j}}{\varepsilon} .
$$

This is a system of ODEs similar to the form

$$
y^{\prime \prime}+\alpha y=\beta,
$$

which has the solution of the form $(\alpha>0)$

$$
y(t)=A e^{i t \sqrt{\alpha}}+B e^{-i t \sqrt{\alpha}}+\frac{\beta}{\alpha} .
$$

Note that all $\lambda_{j}>0(j>0)$ so it is known that the $v_{j}$ functions in the problem have the form

$$
v_{j}(t)=A_{j} e^{\frac{i t \sqrt{\lambda_{j}}}{\varepsilon}}+B_{j} e^{\frac{-i t \sqrt{\lambda_{j}}}{\varepsilon}}+r_{j}, \quad r_{j}=\frac{\varepsilon p \cdot a_{j}}{\lambda_{j}},
$$

and the special $v_{0}$ is given by

$$
v_{0}(t)=\frac{p \cdot a_{0}}{2 \varepsilon} t^{2}+C t+D=C t+D \quad \text { since } a_{0}=0
$$

By plugging the general solution (3.12) into the initial conditions of (3.4), we can formulate equations for $A_{j}$ and $B_{j}(j>0)$,

$$
\begin{gathered}
v(0, x)=0 \Rightarrow \sum_{j=0}^{\infty} v_{j}(0) w_{j}^{\varepsilon}(x)=0 \Rightarrow v_{j}(0)=0 \Rightarrow A_{j}+B_{j}+r_{j}=0 ; \\
v_{t}(0, x)=0 \Rightarrow \sum_{j=0}^{\infty} v_{j}^{\prime}(0) w_{j}^{\varepsilon}(x)=0 \Rightarrow v_{j}^{\prime}(0)=0 \Rightarrow \frac{i \sqrt{\lambda_{j}}}{\varepsilon} A_{j}-\frac{i \sqrt{\lambda_{j}}}{\varepsilon} B_{j}=0 .
\end{gathered}
$$


Similary, for $v_{0}(t)$

$$
v_{0}(0)=0 \Rightarrow C=0, \quad \text { and } \quad v_{0}^{\prime}(0)=0 \Rightarrow D=0,
$$

thus $v_{0}(t) \equiv 0$. We solve for $A_{j}$ and $B_{j}$ and obtain

$$
A_{j}=B_{j}=\frac{r_{j}}{2}=-\frac{\varepsilon p \cdot a_{j}}{2 \lambda_{j}}, \quad j=1,2, \ldots
$$

All in all, the $v_{j}(t)$ coefficients in explicit form are

$$
\left\{\begin{array}{l}
v_{0}(t)=0 \\
v_{j}(t)=-\frac{\varepsilon p \cdot a_{j}}{2 \lambda_{j}}\left(e^{\frac{i t \sqrt{\lambda_{j}}}{\varepsilon}}+e^{\frac{-i t \sqrt{\lambda_{j}}}{\varepsilon}}\right)+\frac{\varepsilon p \cdot a_{j}}{\lambda_{j}}=\frac{\varepsilon p \cdot a_{j}}{\lambda_{j}}\left(1-\cos \frac{t \sqrt{\lambda_{j}}}{\varepsilon}\right) \quad j=1,2, \ldots
\end{array}\right.
$$

The solution to our problem (3.4) can then be expressed as

$$
v(y, t)=\varepsilon p \cdot \sum_{j=1}^{\infty} \frac{a_{j}}{\lambda_{j}}\left(1-\cos \frac{t \sqrt{\lambda_{j}}}{\varepsilon}\right) w_{j}(y) .
$$

Step 5. Now plug the expression (3.19) into the expression (3.3)

$$
f^{\varepsilon}=\left(\nabla u^{\varepsilon}\right) A(x / \varepsilon)=p \cdot\left(1+\sum_{j=1}^{\infty} \frac{a_{j}}{\lambda_{j}}\left(1-\cos \frac{t \sqrt{\lambda_{j}}}{\varepsilon}\right) \nabla_{y} \cdot w_{j}(x / \varepsilon)\right) A(x / \varepsilon) .
$$

We write down and analyze the function $f^{\varepsilon}$ in two parts $f^{\varepsilon}=p \cdot\left(\Lambda_{1}+\Lambda_{2}\right)$, where

$$
\left\{\begin{array}{l}
\Lambda_{1}(x / \varepsilon)=\left(I+\sum_{j=1}^{\infty} \frac{a_{j}}{\lambda_{j}} \nabla_{y} \cdot w_{j}(x / \varepsilon)\right) A(x / \varepsilon) \\
\Lambda_{2}(x / \varepsilon, t)=-\sum_{j=1}^{\infty} \frac{a_{j}}{\lambda_{j}} \cos \frac{t \sqrt{\lambda_{j}}}{\varepsilon} \nabla_{y} \cdot w_{j}(x / \varepsilon) A(x / \varepsilon)
\end{array}\right.
$$

Step $6 a$. First we show that $\Lambda_{1}=\bar{A}$. To do that we need to use the so-called cell problem (or corrector problem, see [11, section 4.5.4]),

$$
\begin{cases}L(y) \chi=-\nabla_{y} \cdot A, & Y \\ \chi & Y \text {-periodic. }\end{cases}
$$

We rewrite the cell problem (3.22) using a eigenfunction expansion

$$
\sum_{j=0}^{\infty} \lambda_{j} \chi_{j} w_{j}=-\sum_{j=1}^{\infty} a_{j} w_{j} \Rightarrow \chi_{j}=-\frac{a_{j}}{\lambda_{j}}, \quad j=1,2, \ldots
$$

where $\chi_{j}$ are column vectors with coefficients of $\chi$ in the eigenfunction expansion. For the other term $\Lambda_{1}$ we now will make good use of the eigenfunction expansion of the 
cell solution $\chi$,

$$
\begin{aligned}
\iint K_{\tau}(t) K_{\eta}(x) \Lambda_{1} \mathrm{~d} x \mathrm{~d} t & =\int K_{\eta}(x)\left(I+\sum_{j=1}^{\infty} \frac{a_{j}}{\lambda_{j}} \nabla_{y} w_{j}(x / \varepsilon)\right) A(x / \varepsilon) \mathrm{d} x \\
& =\int K_{\eta}(x)\left(I-\nabla_{y} \chi(x / \varepsilon)\right) A(x / \varepsilon) \mathrm{d} x \\
& =\int K_{\eta}(x)\left(A(x / \varepsilon)-\nabla_{y} \chi(x / \varepsilon) A(x / \varepsilon)\right) \mathrm{d} x \\
& =\int K_{\eta}(x)\left(A(x / \varepsilon)-A(x / \varepsilon) \nabla_{y} \chi(x / \varepsilon)\right) \mathrm{d} x \\
& =\bar{A}+\mathcal{O}\left(\left(\frac{\varepsilon}{\eta}\right)^{q}\right),
\end{aligned}
$$

where we used Lemma 3.2, in each coordinate direction.

Step $6 b$. Now we should show that

$$
\iint K_{\tau}(t) K_{\eta}\left(x-x_{0}\right) \Lambda_{2} \mathrm{~d} t \rightarrow 0, \quad \frac{\tau}{\varepsilon} \rightarrow \infty .
$$

For that we need a Lemma from [10]:

Lemma 3.2. Let $f^{\varepsilon}(t)=f(t, t / \varepsilon)$, where $f(t, s)$ is 1-periodic in the second variable and $\partial^{r} f(t, s) / \partial t^{r}$ is continuous for $r=0,1, \ldots, p-1$. For any $K \in \mathbb{K}^{p, q}$ there exists constants $C_{1}$ and $C_{2}$, independent of $\varepsilon$ and $\eta$, such that

$$
E=\left|K_{\eta} * f^{\varepsilon}(t)-\bar{f}(t)\right| \leq C_{1} \eta^{p}+C_{2}\left(\frac{\varepsilon}{\eta}\right)^{q}, \quad \bar{f}(t)=\int_{0}^{1} f(t, s) \mathrm{d} s .
$$

If $f=f(t / \varepsilon)$ then we can take $C_{1}=0$. Furthermore, the error is minimized if $\eta$ is chosen to scale with $\varepsilon^{q /(p+q)}$.

We now apply Lemma 3.2 to obtain

$$
\left|\int K_{\tau}(t) \cos \frac{t \sqrt{\lambda_{j}}}{\varepsilon} \mathrm{d} t\right| \leq C_{2}\left(\frac{2 \pi \varepsilon}{\sqrt{\lambda_{j}} \tau}\right)^{q}=C^{\prime} \frac{1}{\lambda_{j}^{q / 2}}\left(\frac{\varepsilon}{\tau}\right)^{q} .
$$

Let $b_{j}$ and the column vector $g(y)$ be defined as

$$
b_{j}=\int K_{\tau}(t) \cos \frac{t \sqrt{\lambda_{j}}}{\varepsilon} \mathrm{d} t, \quad g(y)=\sum_{j=1}^{\infty} b_{j} \chi_{j} w_{j}(y),
$$

where we again used the solution to the cell problem (3.22) in the formulation of $g(y)$. We then express $\iint K_{\tau} K_{\eta} \Lambda_{2} \mathrm{~d} x \mathrm{~d} t$ using $g$, followed by a change of variables:

$$
\begin{aligned}
\iint K_{\tau}(t) K_{\eta}\left(x-x_{0}\right) \Lambda_{2} \mathrm{~d} x \mathrm{~d} t & =-\int K_{\eta} \nabla_{y} \cdot g(x / \varepsilon) A(x / \varepsilon) \mathrm{d} x \\
& =-\int K(x) \nabla_{y} \cdot g\left(\frac{\eta x+x_{0}}{\varepsilon}\right) A\left(\frac{\eta x+x_{0}}{\varepsilon}\right) \mathrm{d} x \\
& =-\int \frac{\varepsilon}{\eta} K(x) A\left(\frac{\eta x+x_{0}}{\varepsilon}\right) \nabla \cdot g\left(\frac{\eta x+x_{0}}{\varepsilon}\right) \mathrm{d} x .
\end{aligned}
$$


By doing integration by parts, using $K\left(e_{k}\right)=K\left(-e_{k}\right)=0(k=1,2, \ldots, d)$, together with Cauchy-Schwartz inequality, we obtain

$$
\begin{aligned}
& \int_{[-1,1]^{d}} \underbrace{\nabla \cdot\left(\frac{\varepsilon}{\eta} K(x) A\left(\frac{\eta x+x_{0}}{\varepsilon}\right)\right)}_{\mathcal{O}(1) \text { column vector }} g\left(\frac{\eta x+x_{0}}{\varepsilon}\right) \mathrm{d} x \\
& \leq\left(\int_{[-1,1]^{d}}\left(\nabla \cdot\left(\frac{\varepsilon}{\eta} K(x) A\left(\frac{\eta x+x_{0}}{\varepsilon}\right)\right)\right)^{2} \mathrm{~d} x \int_{[-1,1]^{d}} g^{2}\left(\frac{\eta x+x_{0}}{\varepsilon}\right) \mathrm{d} x\right)^{1 / 2},
\end{aligned}
$$

which is bounded by $C\|g\|_{\left(L_{\text {per }}^{2}\right)^{d}}$ where $C$ is independent of $\varepsilon$, and $\eta$. Finally we need to show that $\|g\| \rightarrow 0$. This is done by observing that

$$
\|g\|_{\left(L_{\mathrm{per}}^{2}\right)^{d}}^{2}=\sum_{j=1}^{\infty} b_{j}^{2} \chi_{j}^{2} \leq b_{\max }^{2} \sum_{j=1}^{\infty} \chi_{j}^{2}=b_{\max }^{2}\|\chi\|_{\left(L_{\mathrm{per}}^{2}\right)^{d}}^{2},
$$

where $\left|b_{\max }\right|$ is bounded by,

$$
\frac{C^{\prime}}{\lambda_{1}^{q / 2}}\left(\frac{\varepsilon}{\tau}\right)^{q}
$$

following the computations in (3.26). Then finally, we add our results from the calculations above and obtain

$$
\begin{aligned}
\hat{F}\left(x_{0}, p\right) & =p \cdot \iint K_{\tau}(t) K_{\eta}\left(x-x_{0}\right) f^{\varepsilon} \mathrm{d} x \mathrm{~d} t \\
& =p \cdot \iint K_{\tau}(t) K_{\eta}\left(x-x_{0}\right)\left(\Lambda_{1}(t)+\Lambda_{2}(x / \varepsilon, t)\right) \mathrm{d} x \mathrm{~d} t \\
& =p \cdot\left(\bar{A}+\mathcal{O}\left(\left(\frac{\varepsilon}{\eta}\right)^{q}\right)\right) .
\end{aligned}
$$

This proves the theorem.

Final step. Now we show the error estimate $\left|U_{m}^{n}-\bar{u}\left(x_{m}, t_{n}\right)\right| \leq C(T)\left(H^{2}+(\varepsilon / \eta)^{q}\right)$. We observe that $\tilde{F}$ in the Theorem is of the form

$$
\tilde{F}=\tilde{A}(x) p,
$$

where $\tilde{A}$ is $\varepsilon$-periodic. By $(3.31)$,

$$
|\tilde{A}(x)-\bar{A}| \leq C\left(\frac{\varepsilon}{\eta}\right)^{q} .
$$

By choosing $H=n \varepsilon$ for some integer $n$, we find that the macro scheme (2.3) is a standard second order discretization of the problem

$$
\begin{cases}u_{t t}-\tilde{A}(0) u_{x x}=0, & \Omega \times\{0 \leq t \leq \tau\} \\ u(0, x)=f(x), \quad u_{t}=g, & \Omega \times\{t=0\}\end{cases}
$$


since $\tilde{A}\left(x_{m}\right)=\tilde{A}(m n \varepsilon)=\tilde{A}(0)$ for all $m$. Hence, if $g=0$ (the result is true also for $g \neq 0)$,

$$
u_{m}^{n}=\frac{1}{2}\left(f\left(x_{m}-\sqrt{\tilde{A}(0)} t_{n}\right)+f\left(x_{m}+\sqrt{\tilde{A}(0)} t_{n}\right)\right)+\mathcal{O}\left(H^{2}\right) .
$$

On the other hand, the solution of the homogenized (1.3) with $g=0$ is

$$
\bar{u}\left(x_{m}, t_{n}\right)=\frac{1}{2}\left(f\left(x_{m}-\sqrt{\bar{A}} t_{n}\right)+f\left(x_{m}+\sqrt{\bar{A}} t_{n}\right)\right) .
$$

Therefore we get the error estimate

$$
\begin{aligned}
\left|U_{m}^{n}-\bar{u}\left(x_{m}, t_{n}\right)\right| & \leq \sup _{|t| \leq T}|f(x+\sqrt{\tilde{A}(0)} t)-f(x+\sqrt{\bar{A}} t)|+C(T) H^{2} \\
& \leq\left|f^{\prime}\right|_{\infty} T|\sqrt{\tilde{A}}-\sqrt{\bar{A}}|+C(T) H^{2} \\
& \leq C(T)\left(H^{2}+(\varepsilon / \eta)^{q}\right)
\end{aligned}
$$

for $0 \leq t_{n} \leq T$. This proves the Theorem.

\section{Numerical results}

In this section we show numerical results when applying the HMM process to various problems in one, two and three dimensions. The notation in the experiments in the $d$-dimensional setting $(d=1,2,3)$ is the following: We let $Y=[0,1]^{d}$ denote the macro domain and $\varepsilon$ be the micro problem scale. We denote $H$ and $K$ to be the macro grid size and time step respectively and for the micro-scale we denote $h$ and $k$ to be the grid size and time step respectively. We use explicit second order accurate finite difference schemes (see Appendix).

4.1. Convergence study of different kernels. In figures 4.1 and 4.2 we present convergence results for the flux $F$ in terms of $\eta / \varepsilon$. We use different type of kernels for the problem (4.1) with $A^{\varepsilon}(x)=A_{1}(x / \varepsilon)$ and $A^{\varepsilon}(x)=A_{2}(x, x / \varepsilon)$ where $A_{1}(y)=1.1+\sin (2 \pi y)$ and $A_{2}(x, y)=1.1+\frac{1}{2}(\sin 2 \pi x+\sin 2 \pi y)$. We compare our numerical results to the theoretical bounds in Theorem 3.1. On problems with both fast and slow scales, which is not directly covered by Theorem 3.1, we see a (slow) growth of the error as $\tau, \eta \rightarrow \infty$ consistent with the general approximation result in Lemma 3.2. We plot $(\varepsilon / \eta)^{q}$ and $\eta^{p}$ separately with dashed lines.

4.2. 1D results. The general form for the one-dimensional examples is:

$$
\begin{cases}u_{t t}^{\varepsilon}=\partial_{x} A^{\varepsilon} u_{x}^{\varepsilon}, & Y \times\{0 \leq t \leq T\}, \\ u^{\varepsilon}=f, \quad u_{t}^{\varepsilon}=0, & Y \times\{t=0\},\end{cases}
$$

where $Y=[0,1]$. We show some dynamics in figure. 4.3 where we solved (4.1) for the $A^{\varepsilon}$ and $f$ given in example one below. The homogenized solution to (4.1) will be of the form

$$
\begin{cases}\bar{u}_{t t}=\partial_{x} \bar{A} \bar{u}_{x}, & Y \times\{0 \leq t \leq T\}, \\ \bar{u}=f, \quad \bar{u}_{t}=0, & Y \times\{t=0\},\end{cases}
$$

where $\bar{A}$ is given by the harmonic average of $A(x, y)$ over one $Y$-period,

$$
\bar{A}(x)=\int_{0}^{1} \frac{\mathrm{d} y}{A(x, y)}
$$

and $x$ being held fixed. 

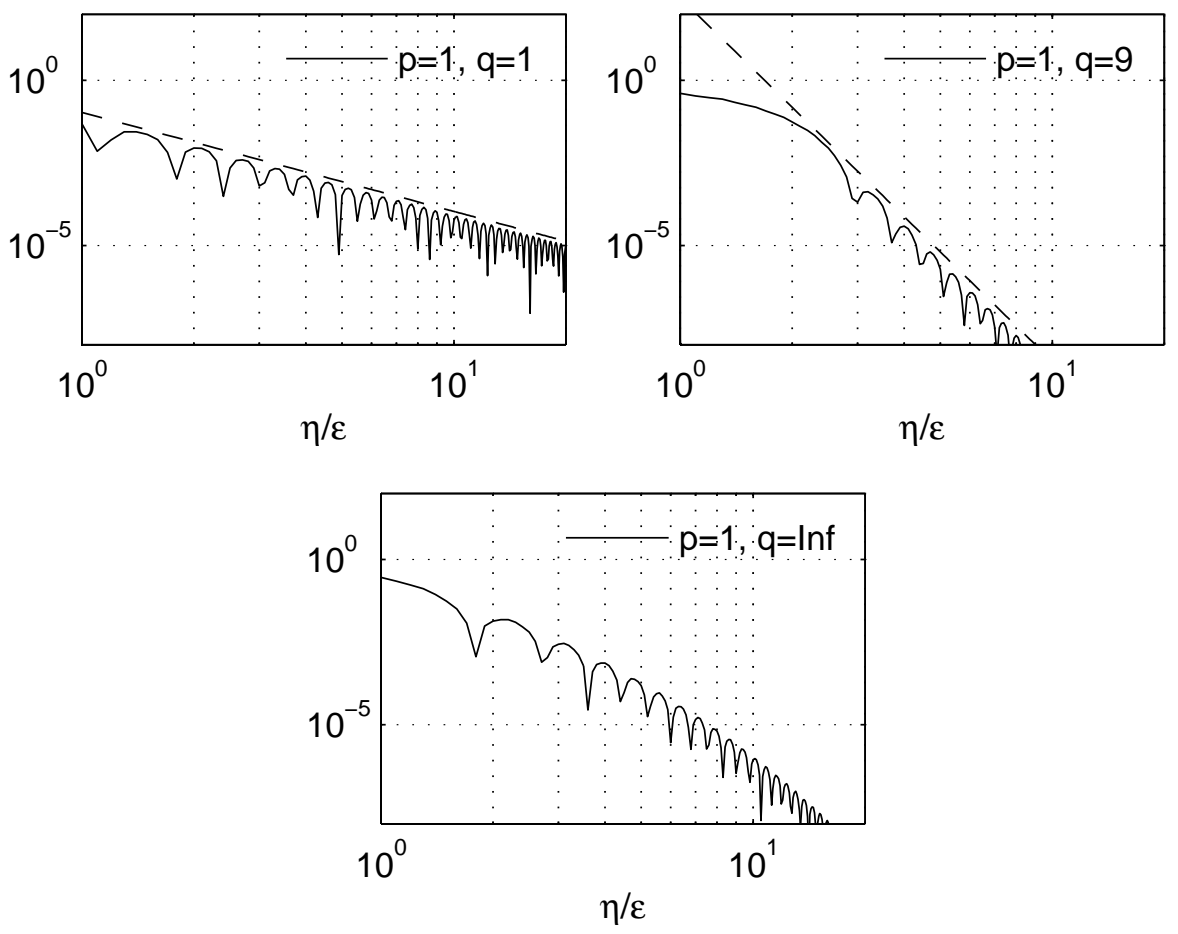

FIG. 4.1. Convergence results, error $|\tilde{F}-\bar{F}|$ plotted against $\eta / \varepsilon(\tau=\eta)$ for fixed $\varepsilon=0.01$ and where $A^{\varepsilon}=A_{1}$ with only fast scales. The dashed line corresponds to the $(\varepsilon / \eta)^{q}$ term in Theorem 3.1. The bottom figure shows results for the exponential kernel (see Remark 2.1) and indicates super algebraic convergence rate.

4.2.1. Example one. In the first wave propagation problem we choose $A^{\varepsilon}$ and $f$ as

$$
\left\{\begin{array}{l}
A^{\varepsilon}(x)=A(x / \varepsilon), \quad A(y)=1.1+\sin 2 \pi y, \\
f(x)=\exp \left(-\left(x-x_{0}\right)^{2} / \sigma^{2}\right), \quad x_{0}=0.5, \quad \sigma=0.1 .
\end{array}\right.
$$

We can compute $\bar{A}$ from (4.3) with techniques from complex analysis

$$
\bar{A}=\sqrt{\frac{21}{100}}=0.458257569495584 \ldots
$$

We will solve (4.2) with a fully resolved discretization or direct numerical simulation (DNS), discretized homogenized solution (HOM), and our HMM method (HMM). We have used $\varepsilon=0.01, \tau=\eta=10 \varepsilon$. In figure 4.4 we show a snapshot of the solutions these methods at time $T=1$. We use a kernel (same in both time and space) $K \in \mathbb{K}^{5,6}$, that is $K$ has 5 zero moments and is 6 times continuously differentiable.

4.2.2. Example two. We now consider a variation of (4.1) where $A^{\varepsilon}$ is defined as

$$
A^{\varepsilon}(x)=A(x, x / \varepsilon), \quad A(x, y)=1.1+\frac{1}{2}(\cos 2 \pi x+\sin 2 \pi y) .
$$



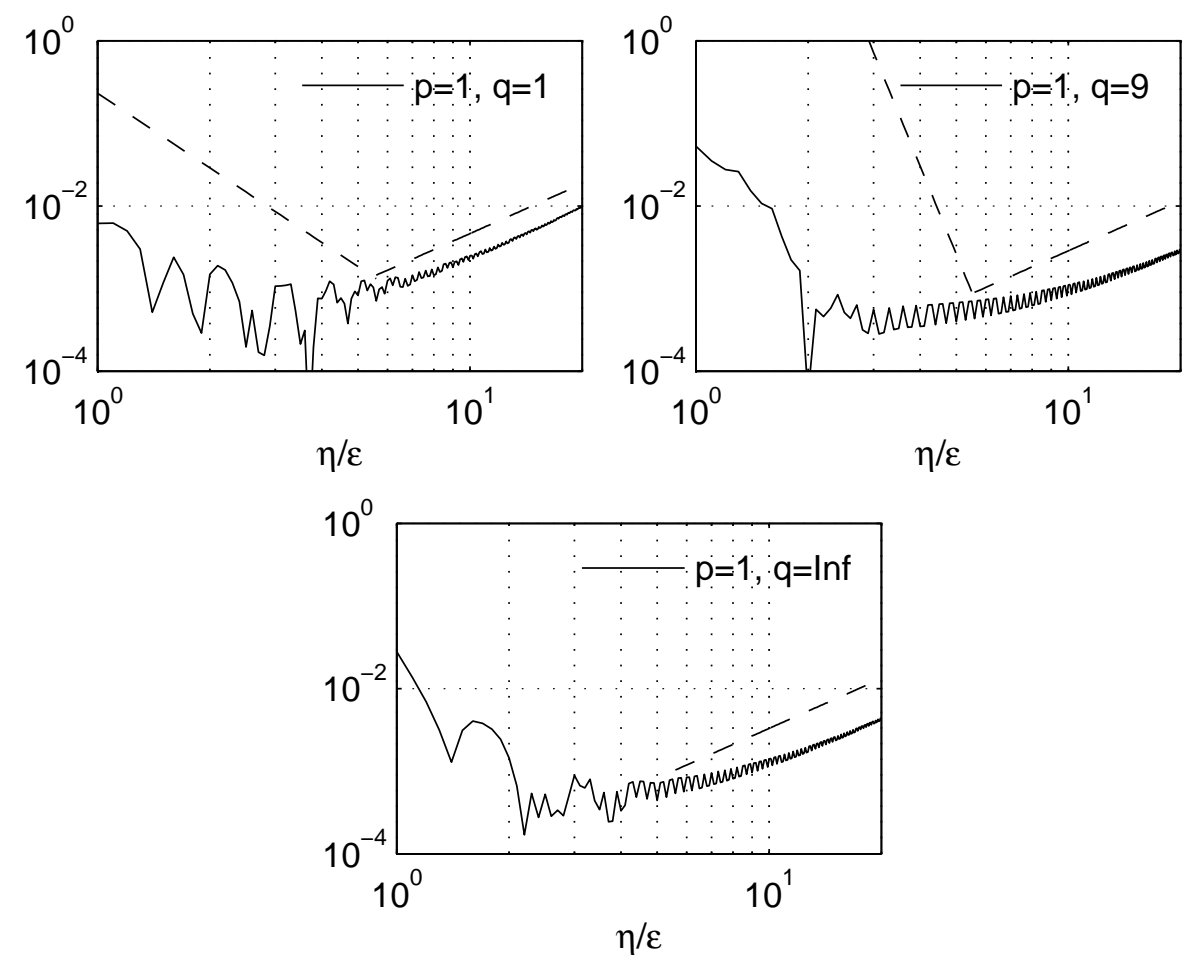

FIG. 4.2. Convergence results, error $|\tilde{F}-\bar{F}|$ plotted against $\eta / \varepsilon(\tau=\eta)$ for fixed $\varepsilon=0.01$ and where $A^{\varepsilon}=A_{2}$ with both fast and slow scales. The dashed line with negative slope corresponds to the theoretical bound from the first term in Lemma 1 and the dashed line with positive slope corresponds to the $\eta^{p}$ term.

The homogenized operator $\bar{A}$ will not be constant but a function with explicit $x$ dependence. We compute analytically $\bar{A}(x)$ to be

$$
\bar{A}(x)=\sqrt{\alpha(x)^{2}-\beta^{2}} \quad \alpha(x)=1.1+\frac{1}{2} \cos 2 \pi x, \quad \beta=\frac{1}{2} .
$$

For this experiment we use $\varepsilon=0.01, K=2 H, H=3.33 \cdot 10^{-3}$. For the micro problem we use $k / h=0.5$ and $h=\varepsilon / 64$. The kernel from $\mathbb{K}^{9,9}$ with $\tau=\eta=10 \varepsilon$. The small $H$ is to lessen the effect of the numerical dispersion. We show results from $T=1$ in figure 4.5 .

4.2.3. Example three. In the last one-dimensional example the macro equation is unknown, i.e. homogenization does not provide $\bar{A}$. We define $A^{\varepsilon}$ as a sum of many micro-scale oscillations

$$
\begin{cases}A^{\varepsilon}(x)=1.1+\frac{1}{5} \sum_{i=1}^{5} \sin 2 \pi \frac{x}{\varepsilon_{i}}, & \varepsilon_{i}=\frac{1}{90+5(i-1)} \\ f(x)=\exp \left(-\left(x-x_{0}\right)^{2} / \sigma^{2}\right), & x_{0}=0.5, \quad \sigma=0.1 .\end{cases}
$$

A plot of $A^{\varepsilon}$ is shown in figure 4.6. The numerical parameters for the macro-solver (HMM and homogenized) are $H=3.33 \cdot 10^{-3}, K=0.5 H$. The micro solver uses $\tau=$ 

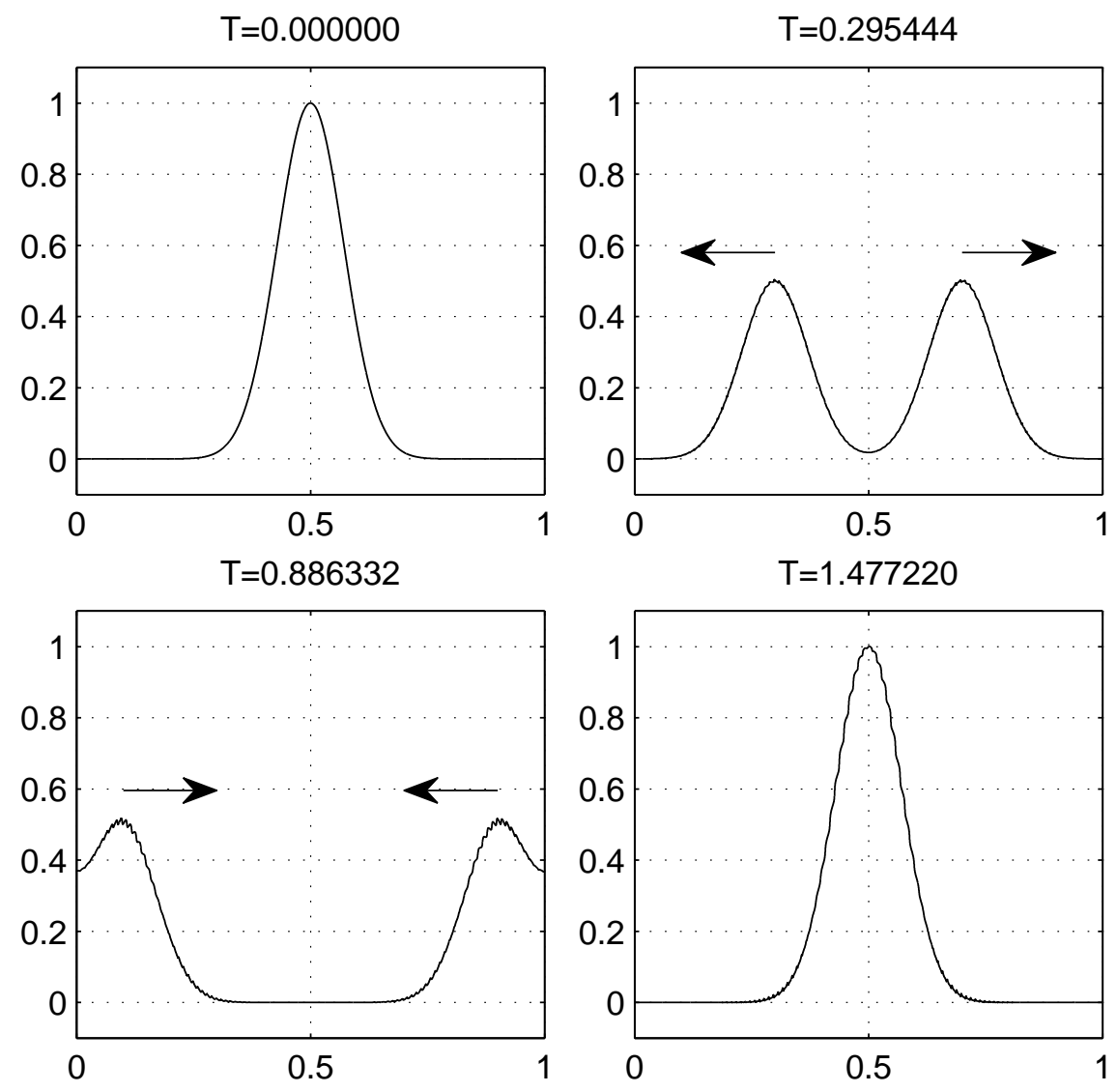

FIG. 4.3. The dynamics of the problem (4.1) can be seen for 4 snapshots at $t_{i}=3 /(i \sqrt{\bar{A}}), 0 \leq i \leq$ 3. Note the small oscillations superimposed on the smooth profile. We observe how the initial pulse separates in one left going and one right going pulse. The effect of the periodic boundary condition can be seen as the waves pass each other at the boundaries between frames 2 and 3.
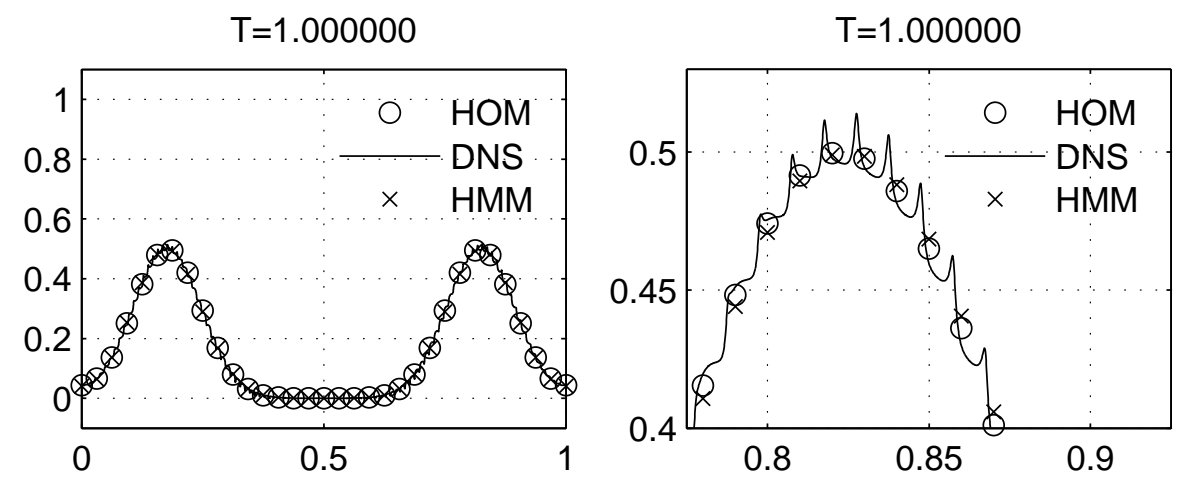

FIG. 4.4. A snapshot of two superimposed solutions to (4.1) together with a zoomed section. 

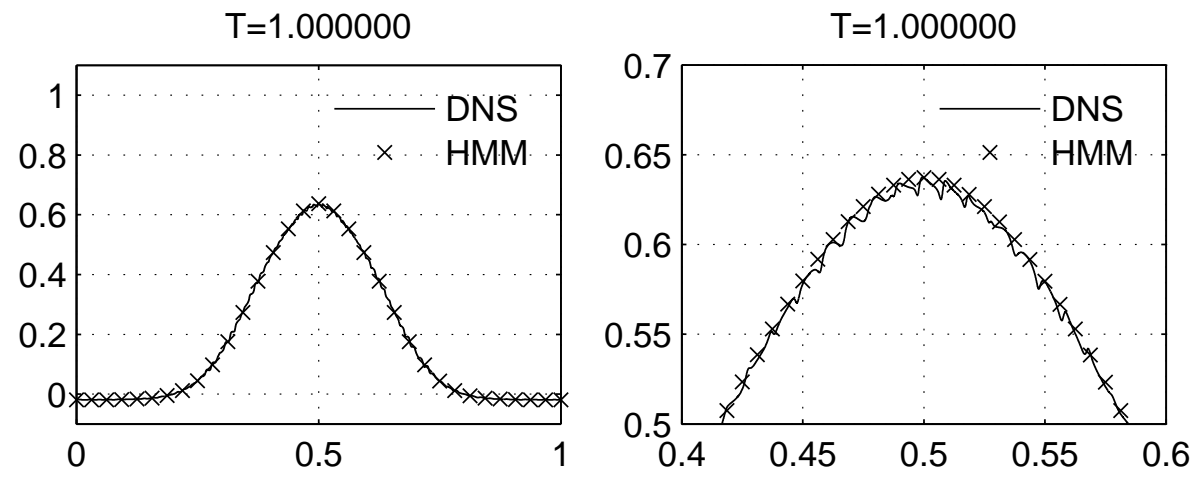

FIG. 4.5. A snapshot of two superimposed solutions to (4.6) together with a zoomed section.
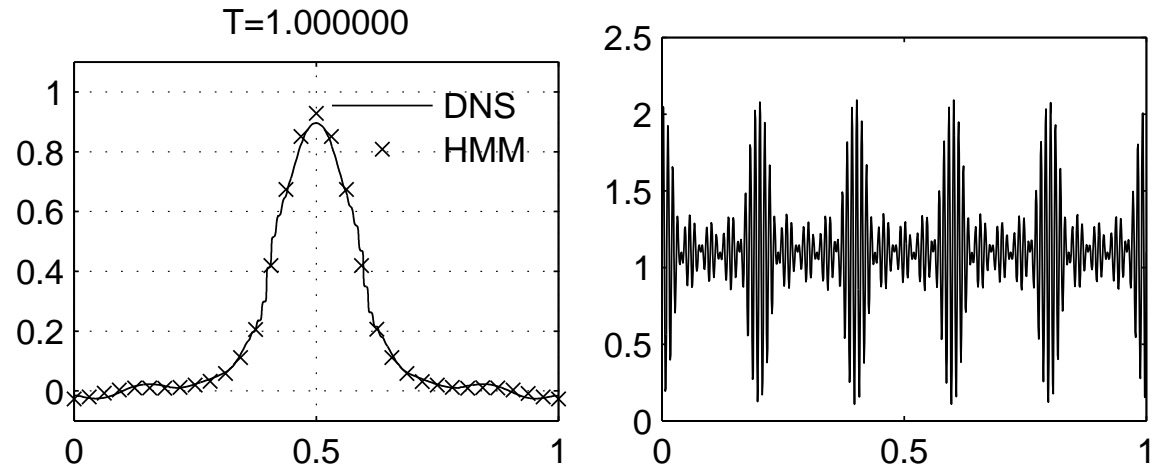

FIG. 4.6. A snapshot of a direction solution to (4.6) and the HMM solution (left) together with the material coefficient $A^{\varepsilon}$ from example 4.2.3 (right).

$10 \varepsilon_{3}, \eta=\varepsilon_{3}, h=\varepsilon_{3} / 64$ and $k=0.5 h$. The kernel $K$ used, for both time and space, is $K \in \mathbb{K}^{5,6}$. The results are shown in figure 4.6.

4.3. 2D results. In this section we present the numerical results for a two dimensional wave propagation problem over the unit square $Y=[0,1] \times[0,1]$.

4.3.1. Example four. We define $A^{\varepsilon}(x)$ by the diagonal matrix,

$$
\left\{\begin{array}{l}
A^{\varepsilon}(x)=\operatorname{diag}\left(a^{\varepsilon}(x), a^{\varepsilon}(x)\right) \\
a^{\varepsilon}(x)=a(x / \varepsilon), \quad a(y)=1.1+\sin 2 \pi y_{1} .
\end{array}\right.
$$

The corresponding homogenized matrix $\bar{A}$ in (1.3),

$$
\bar{A}=\operatorname{diag}(\sqrt{0.21}, 1.1),
$$

and as in 1D the initial data $f$ is defined as a Gaussian,

$$
\left\{\begin{array}{l}
f(x)=\exp \left(-\left\|x-x_{0}\right\|_{2}^{2} / \sigma^{2}\right), \\
x_{0}=\left[\begin{array}{ll}
0.5 & 0.5
\end{array}\right], \quad \sigma=0.1 .
\end{array}\right.
$$




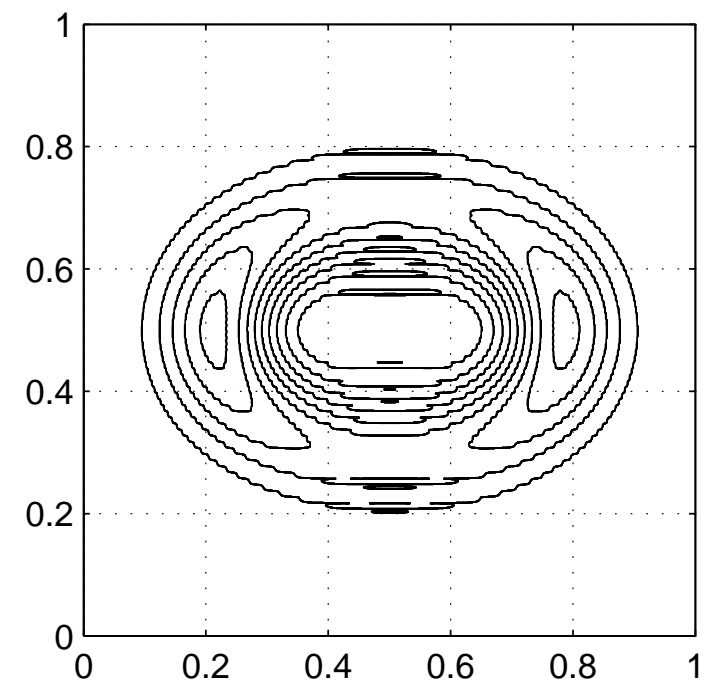

FIG. 4.7. Full numerical simulation when $A^{\varepsilon}$ has only a fast scale.

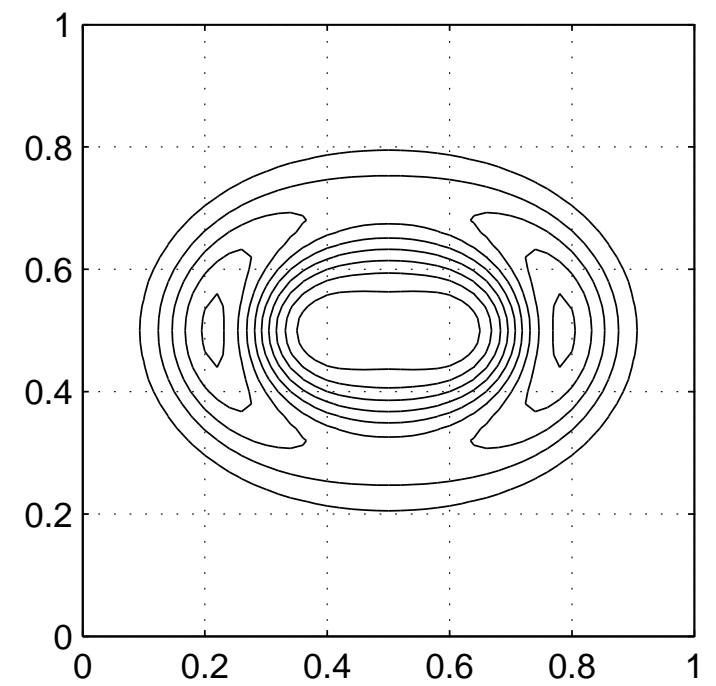

FIG. 4.8. Direct solution of the homogenized equation when $\bar{A}$ is constant.

We use the exponential kernel $K_{\exp } \in \mathbb{K}^{1, \infty}$. We let $T=1$ and the scale parameter $\varepsilon$ is set to 0.01 . The macro scheme uses $H=3.33 \cdot 10^{-3}$ and $K=0.5 H$. The micro scheme uses $h=\varepsilon / 64$ and $k=0.5 h$. We show the numerical results in figures 4.7, 4.8, and 4.9.

4.3.2. Example five. We let $A^{\varepsilon}(x)$ be defined by the diagonal matrix,

$$
\left\{\begin{array}{l}
A^{\varepsilon}(x)=\operatorname{diag}\left(a^{\varepsilon}(x), a^{\varepsilon}(x)\right) \\
a^{\varepsilon}(x)=a(x, x / \varepsilon), \quad a(x, y)=1.1+\frac{1}{2}\left(\sin 2 \pi x_{1}+\sin 2 \pi y_{1}\right) .
\end{array}\right.
$$




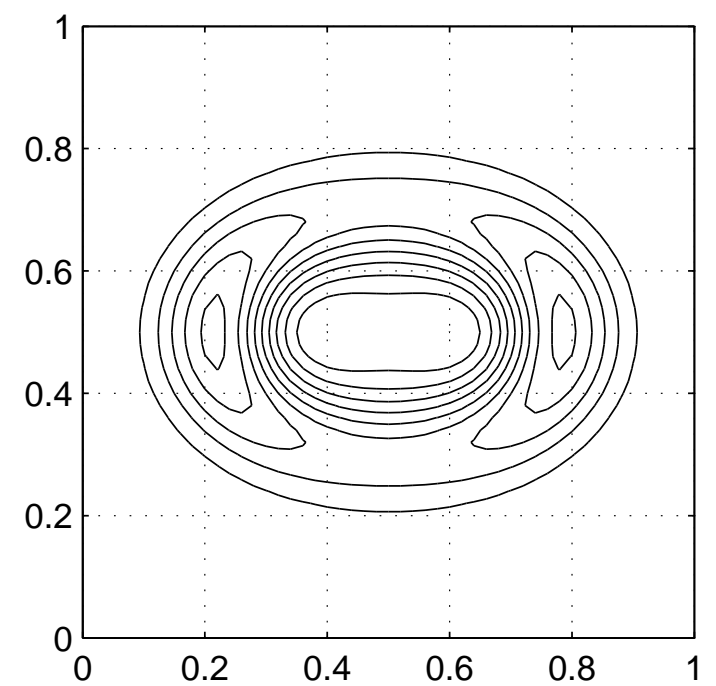

FIG. 4.9. HMM approach, when $A^{\varepsilon}$ has only fast scales.

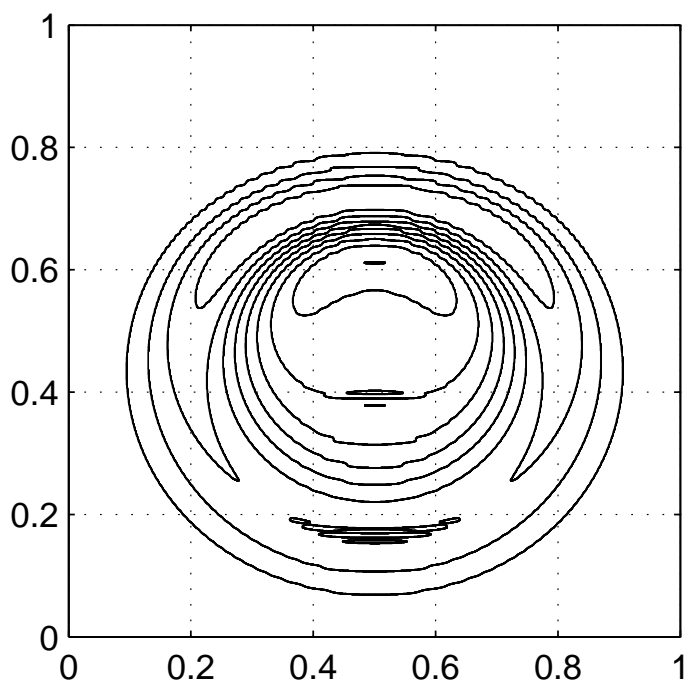

FIG. 4.10. Full numerical simulation and $A^{\varepsilon}$ is defined by (4.12).

and the corresponding homogenized matrix $\bar{A}$ in (1.3),

$$
\left\{\begin{array}{l}
\bar{A}(x)=\operatorname{diag}(\bar{a}(x), 1.1), \\
\bar{a}(x)=\sqrt{\alpha(x)^{2}-\beta^{2}}, \quad \alpha(x)=1.1+0.5 \sin 2 \pi x_{1}, \quad \beta=0.5 .
\end{array}\right.
$$

The numerical parameters are chosen the same as in example 4.3.1. We show the numerical results in figures 4.10 and 4.11 .

4.4. 3D results. Here we present numerical results for a wave propagation problem in three dimensions in a locally periodic media over the box $Y=[0,1]^{3}$. 


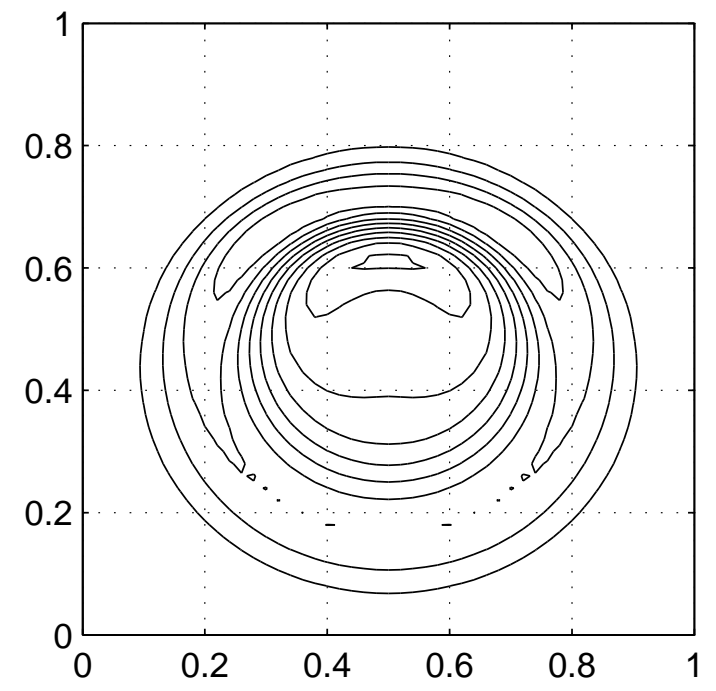

FIG. 4.11. HMM approach and $A^{\varepsilon}$ is defined by (4.12).

4.4.1. Example six. In this three dimensional problem $A^{\varepsilon}(x)$ is a diagonal matrix

$$
\left\{\begin{array}{l}
A^{\varepsilon}(x)=\operatorname{diag}\left(a^{\varepsilon}(x), a^{\varepsilon}(x), a^{\varepsilon}(x)\right), \\
a^{\varepsilon}(x)=a(x / \varepsilon), \quad a(y)=1.1+\sin 2 \pi y_{1},
\end{array}\right.
$$

the corresponding homogenized matrix $\bar{A}$ in (1.3) is

$$
\bar{A}(x)=\operatorname{diag}(\sqrt{0.21}, 1.1,1.1),
$$

and the initial data $f$ is defined as a Gaussian,

$$
\left\{\begin{array}{l}
f(x)=\exp \left(-\left\|x-x_{0}\right\|_{2}^{2} / \sigma^{2}\right) \\
x_{0}=\left[\begin{array}{lll}
0.5 & 0.5 & 0.5
\end{array}\right], \quad \sigma=0.1 .
\end{array}\right.
$$

In this experiment we have used $\varepsilon=0.01$. The homogenized simulation uses $T=0.25$, $H=0.05, K=0.25 H$. The HMM solver uses $T=0.25, H=0.05, K=0.25 H$ on the macro solver. The micro solver uses $\eta=\varepsilon, \tau=5 \varepsilon, h=\varepsilon / 64, k=0.3 h$ and a polynomial kernel $K \in \mathbb{K}^{9,9}$. The results are presented in figure 4.12.

REMARK 4.1. Due to the vast computational expense to use DNS we are unable to show DNS results.

4.5. Long time example. We finally show a problem of the same form as example 4.2.1, but we will solve it for $T=\mathcal{O}\left(\varepsilon^{-2}\right)$. In [20] it was shown that the effective equation in this long time regime is of the form,

$$
\begin{cases}u_{t t}-\bar{A} u_{x x}-\beta \varepsilon^{2} u_{x x x x}=0, & Y \times\{0 \leq t \leq T\}, \\ u=f, \quad u_{t}=0, & Y \times\{t=0\} .\end{cases}
$$



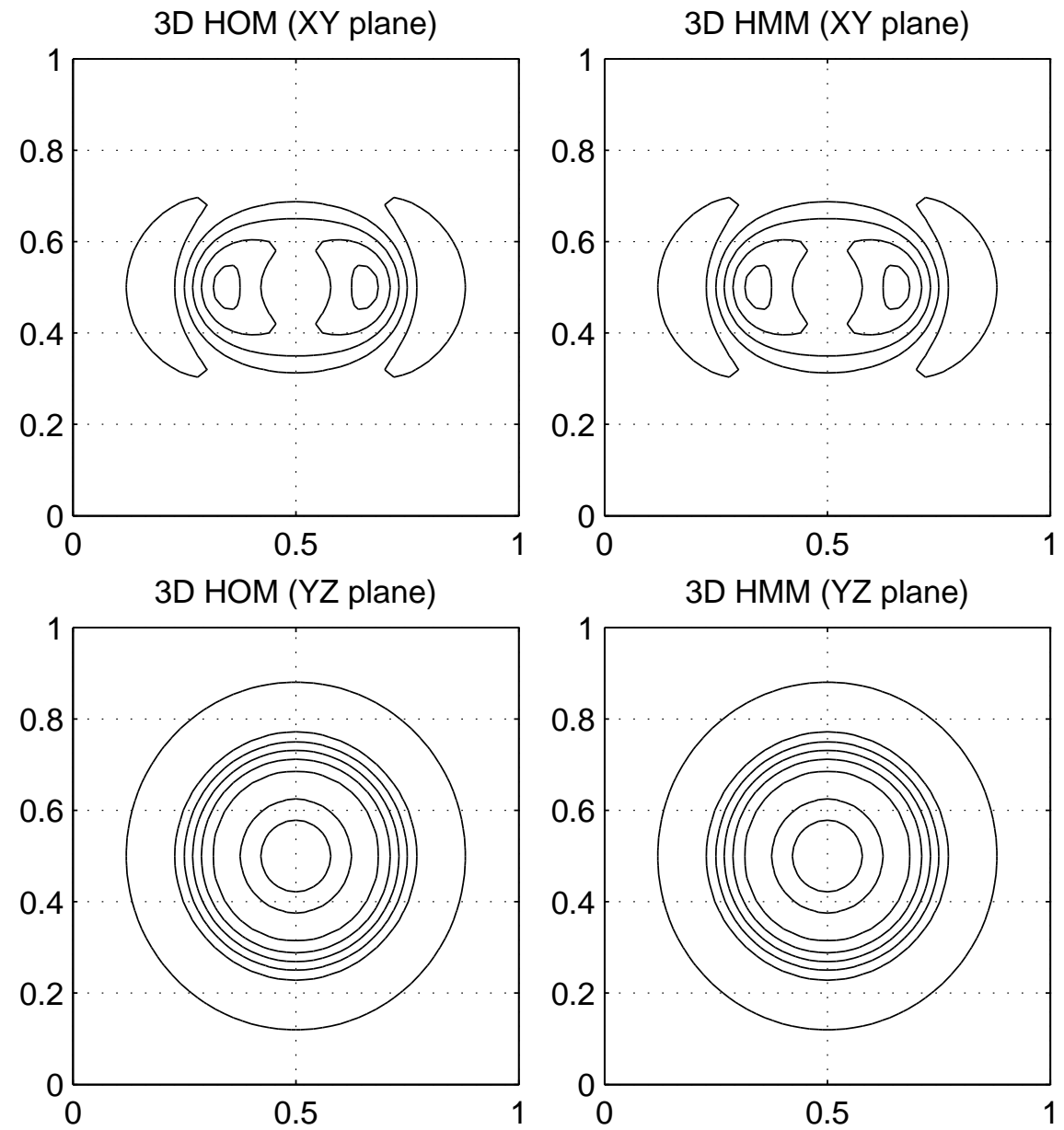

Fig. 4.12. Three dimensional solutions of the homogenized equation and by using the HMM technique.

This is still on the same flux form as assumed in (2.1) with $F=\bar{A} u_{x}+\beta \varepsilon^{2} u_{x x x}$. Therefore, it turns out that we only need to make the HMM process a little bit more accurate for long time computations. The modifications needed are:

- Initial data in micro solver needs to be of higher order. We use a third order polynomial to approximate the higher macro derivatives.

- The integration kernel needs to be smoother to give more accurate $F$ (error less than $\mathcal{O}\left(\varepsilon^{2}\right)$ ) in order to capture the correct dispersion relationship, i.e., $(\varepsilon / \eta)^{q}<\varepsilon^{2}$. This implies also that:

- The micro box needs to be a little bigger, $\tau, \eta \sim \varepsilon^{1-2 / q}$, where $q$ is defined in (2.1).

We present the numerical computations in figure 4.13 for the same $A$ and initial data as in the first example and $\varepsilon=0.01, T=91.58$ (approximately $62 \sqrt{\bar{A}}$ ). The HMM solver uses $H=1 / 300$ (approximately $31 / \sqrt{T}$ ), $K=H / 2$ and a kernel with $\tau=\eta=10 \varepsilon$ from $\mathbb{K}^{5,6}$. The micro solver uses $h=\varepsilon / 64$ and $k=h / 2$ and the long time 
DNS computation itself uses $h=\varepsilon / 128, k=h / 2$.

The long time problem is part of ongoing research by the authors and we will shed some more light on it in a future publication.
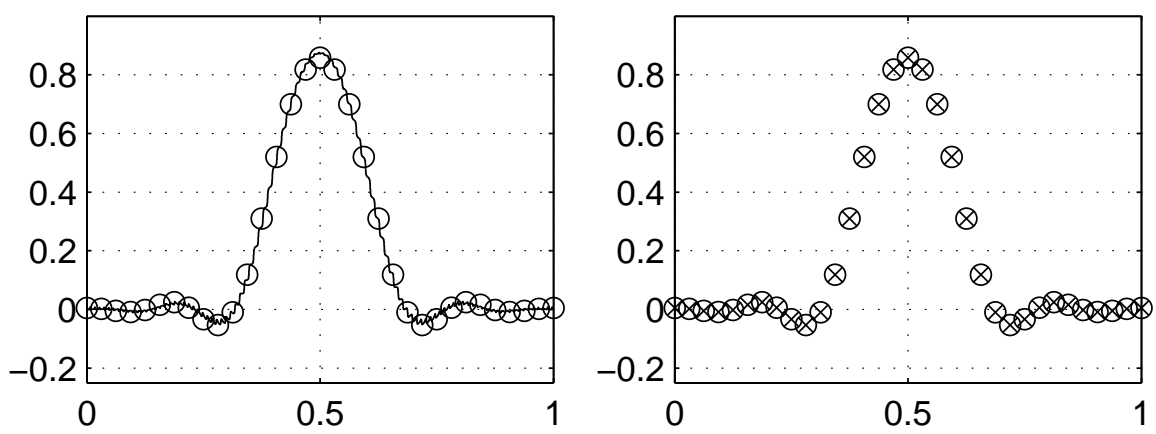

FIG. 4.13. $1 D$ longtime DNS simulation (thin line) compared to a finite difference solution of the effective equation (4.17) (circles) and a HMM solution (crosses).

\section{Conclusions}

We have developed and analyzed numerical methods for multi-scale wave equations with oscillatory coefficients. The methods are based on the framework of the heterogeneous multi-scale method (HMM) and have substantially lower computational complexity than standard discretization algorithms. Convergence proofs for finite time approximation are presented in the case of periodic coefficients in multiple dimensions. Numerical experiments in one, two, and three spatial dimensions show the accuracy and efficiency of the new techniques. Finally we explored simulation over very long time intervals. The effective equation for very long time is different from the finite time homogenized equation. Dispersive effects enter, and the effective equation must be modified [20]. It is interesting to note that our HMM approach with just minor modifications accurately captures these dispersive phenomena.

\section{Appendix A. Numerical schemes.}

We present a detailed description of the numerical schemes used in the macro and micro solvers. The schemes are designed for one, two, and three dimensions and can be generalized to higher dimensions. All the schemes are second order accurate in both time and space.

A.1. 1D equation. The finite difference scheme on the macro level has the form

$$
\left\{\begin{array}{l}
U_{m}^{n+1}=2 U_{m}^{n}-U_{m}^{n-1}+K^{2} Y_{m}^{n} \\
Y_{m}^{n}=\frac{1}{H}\left(F_{m+\frac{1}{2}}^{n}-F_{m-\frac{1}{2}}^{n}\right) \\
F_{m \pm 1 / 2}^{n}=F\left(x_{m \pm 1 / 2}, P_{m \pm 1 / 2}^{n}\right)
\end{array}\right.
$$

where $P_{m-1 / 2}^{n}=\frac{1}{H}\left(U_{m}^{n}-U_{m-1}^{n}\right)$ and $P_{m+1 / 2}^{n}=\frac{1}{H}\left(U_{m+1}^{n}-U_{m}^{n}\right)$. The micro level 
scheme defined analogously:

$$
\left\{\begin{array}{l}
u_{m}^{n+1}=2 u_{m}^{n}-u_{m}^{n-1}+k^{2} y_{m}^{n} \\
y_{m}^{n}=\frac{1}{h}\left(f_{m+1 / 2}^{n}-f_{m-1 / 2}^{n}\right) \\
f_{m+1 / 2}^{n}=a_{m+\frac{1}{2}} \frac{u_{m+1}^{n}-u_{m}^{n}}{h} \\
f_{m-1 / 2}^{n}=a_{m-\frac{1}{2}} \frac{u_{m}^{n}-u_{m-1}^{n}}{h}
\end{array}\right.
$$

A.2. 2D equation. The two dimensional problem is discretized with a scheme with the following schemes: The finite difference scheme on the macro level is

$$
\left\{\begin{array}{l}
U_{m}^{n+1}=2 U_{m}^{n}-U_{m}^{n-1}+K^{2} Y_{m}^{n} \\
Y_{m}^{n}=\frac{1}{H}\left(F_{m+\frac{1}{2} e_{1}}^{(1)}-F_{m-\frac{1}{2} e_{1}}^{(1)}\right)+\frac{1}{H}\left(F_{m+\frac{1}{2} e_{2}}^{(2)}-F_{m-\frac{1}{2} e_{2}}^{(2)}\right) \\
F_{m \pm \frac{1}{2} e_{k}}^{(k)}=F\left(x_{m \pm \frac{1}{2} e_{k}}, P_{m \pm \frac{1}{2} e_{k}}^{n}\right), \quad t=t_{n}
\end{array}\right.
$$

where $P_{m+\frac{1}{2} e_{2}}^{n}$ is given by (see figure 2.1)

$$
P_{m+\frac{1}{2} e_{2}}^{n}=\left[\frac{1}{2 H}\left(\frac{U_{m+e_{1}}+U_{m+e_{1}+e_{2}}}{2}-\frac{U_{m-e_{1}}+U_{m-e_{1}+e_{2}}}{2}\right) \frac{1}{H}\left(U_{m+e_{2}}-U_{m}\right)\right],
$$

and the other $P_{m \pm \frac{1}{2} e_{k}}^{n}$ are components defined analogously. The micro level scheme is formulated as

$$
\left\{\begin{array}{l}
u_{m}^{n+1}=2 u_{m}^{n}-u_{m}^{n-1}+k^{2} y_{m}^{n} \\
y_{m}^{n}=\frac{1}{h}\left(f_{m+\frac{1}{2} e_{1}}^{(1)}-f_{m-\frac{1}{2} e_{1}}^{(1)}\right)+\frac{1}{h}\left(f_{m+\frac{1}{2} e_{2}}^{(2)}-f_{m-\frac{1}{2} e_{2}}^{(2)}\right) \\
f_{m+\frac{1}{2} e_{1}}^{(1)}=\frac{a_{m+\frac{1}{2} e_{1}}^{(11)}}{h}\left(u_{m+e_{1}}^{n}-u_{m}^{n}\right)+\frac{a_{m+\frac{1}{2} e_{1}}^{(12)}}{2 h}\left(\frac{u_{m+e_{2}}^{n}+u_{m+e_{1}+e_{2}}^{n}}{2}-\frac{u_{m-e_{2}}^{n}+u_{m+e_{1}-e_{2}}^{n}}{2}\right) \\
f_{m-\frac{1}{2} e_{1}}^{(1)}=\frac{a_{m-\frac{1}{2} e_{1}}^{(11)}}{h}\left(u_{m}^{n}-u_{m-e_{1}}^{n}\right)+\frac{a_{m-\frac{1}{2} e_{1}}^{(12)}}{2 h}\left(\frac{u_{m+e_{2}}^{n}+u_{m-e_{1}+e_{2}}^{n}}{2}-\frac{u_{m-e_{2}}^{n}+u_{m-e_{1}-e_{2}}^{n}}{2}\right) \\
f_{m+\frac{1}{2} e_{2}}^{(2)}=\frac{a_{m+\frac{1}{2} e_{2}}^{(21)}}{2 h}\left(\frac{\left.u_{m+e_{1}}^{n}+u_{m+e_{1}+e_{2}}^{n}-\frac{u_{m-e_{1}}^{n}+u_{m-e_{1}+e_{2}}^{n}}{2}\right)+\frac{a_{m+\frac{1}{2} e_{2}}^{(22)}}{h}\left(u_{m+e_{2}}^{n}-u_{m}^{n}\right)}{2}\right)+\frac{a_{m-\frac{1}{2} e_{2}}^{(22)}}{h}\left(u_{m}^{n}-u_{m-e_{2}}^{n}\right) . \\
f_{m-\frac{1}{2} e_{2}}^{(2)}=\frac{a_{m-\frac{1}{2} e_{2}}^{(21)}}{2 h}\left(\frac{u_{m+e_{1}}^{n}+u_{m+e_{1}-e_{2}}^{n}}{2}-\frac{u_{m-e_{1}}^{n}+u_{m-e_{1}-e_{2}}^{n}}{2}\right)
\end{array}\right.
$$

When approximating $f_{m-\frac{1}{2} e_{1}}^{(2)}$ we take the average of $u_{m \pm e_{2}}^{n}$ and $u_{m+e_{1} \pm e_{2}}^{n}$ to approximate $u\left(x_{m+\frac{1}{2} e_{1} \pm e_{2}}, t^{n}\right)$. Then we use those two averages to approximate the $y$ derivate of $u$ at $u\left(x_{m-\frac{1}{2} e_{1}}\right)$. The scheme is second order in both space and time.

A.3. 3D equation. The macro scheme for the three dimensional problem is of the form

$$
\left\{\begin{array}{l}
U_{m}^{n}=2 U_{m}^{n}-U_{m}^{n-1}+K^{2} Y_{m}^{n}, \\
Y_{m}^{n}=\frac{1}{H}\left(F_{m+\frac{1}{2} e_{1}}^{(1)}-F_{m-\frac{1}{2} e_{1}}^{(1)}\right)+\frac{1}{H}\left(F_{m+\frac{1}{2} e_{2}}^{(2)}-F_{m-\frac{1}{2} e_{2}}^{(2)}\right)+\frac{1}{H}\left(F_{m+\frac{1}{2} e_{3}}^{(3)}-F_{m-\frac{1}{2} e_{3}}^{(3)}\right) \\
F_{m \pm \frac{1}{2} e_{k}}^{(k)}=F\left(x_{m \pm \frac{1}{2} e_{k}}, P_{m \pm \frac{1}{2} e_{k}}^{n}\right), \quad t=t_{n},
\end{array}\right.
$$


where $P_{m+\frac{1}{2} e_{3}}^{n}$ is defined as,

$$
P_{m+\frac{1}{2} e_{3}}^{n}=\left[\begin{array}{c}
\frac{1}{2 H}\left(\frac{U_{m+e_{1}}+U_{m+e_{1}+e_{3}}}{2}-\frac{U_{m-e_{1}}+U_{m-e_{1}+e_{3}}}{2}\right) \\
\frac{1}{2 H}\left(\frac{U_{m+e_{2}}+U_{m+e_{2}+e_{3}}}{2}-\frac{U_{m-e_{2}}+U_{m-e_{2}+e_{3}}}{2}\right) \\
\frac{1}{H}\left(U_{m+e_{3}}-U_{m}\right)
\end{array}\right],
$$

and the other $P_{m \pm \frac{1}{2} e_{k}}^{n}$ defined analogously. The micro level scheme is a second order accurate scheme defined analogous with the $2 \mathrm{D}$ scheme (A.5)

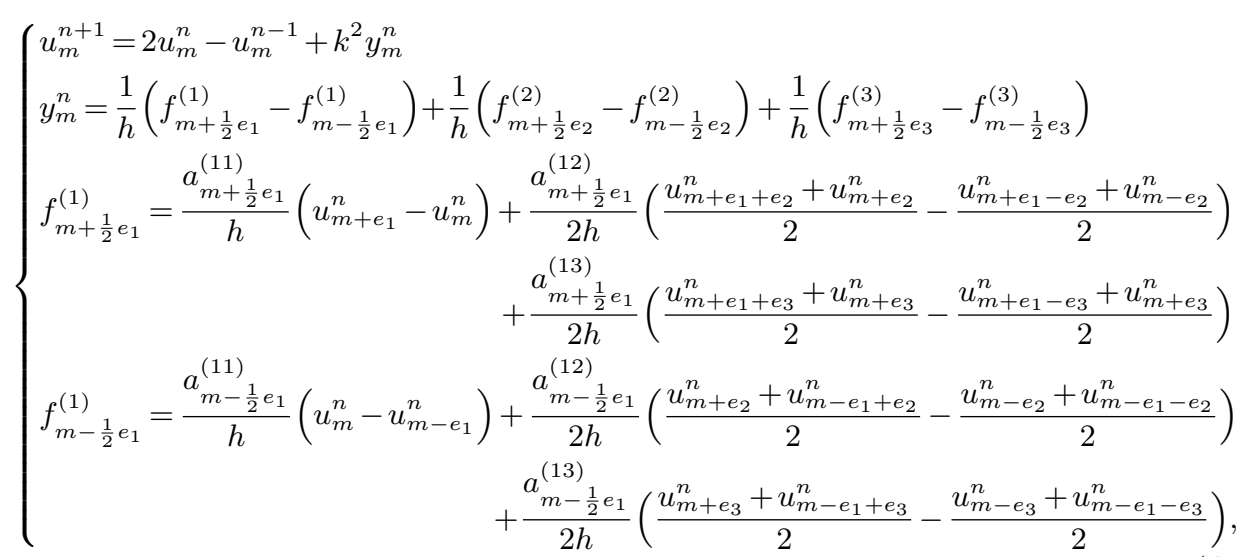

and the other terms as,

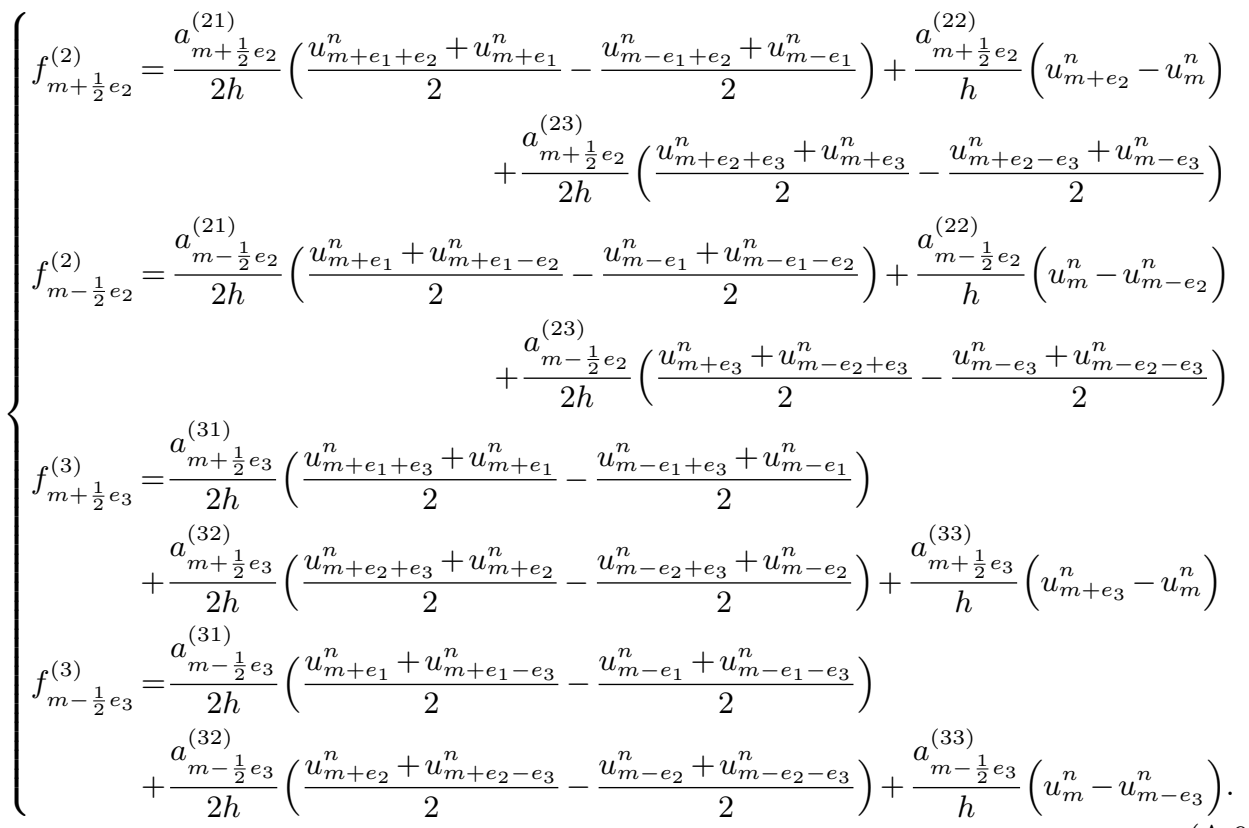




\section{REFERENCES}

[1] A. Abdulle and W. E, Finite difference heterogeneous multi-scale method for homogenization problems, J. Comput. Phys., 191, 18-39, 2003.

[2] A. Bensoussan, J.L. Lions and G. Papanicolaou, Asymptotic Analysis in Periodic Structures, North-Holland Pub. Co., 1978.

[3] D. Cioranescu and P. Donato, An Introduction to Homogenization, in Oxford Lecture Series in Mathematics and its Applications, Oxford University Press Inc., 17, 1999.

[4] W. E and B. Engquist, The heterogeneous multiscale methods, Commun. Math. Sci., 87-133, 2003.

[5] W. E, B. Engquist and Z. Huang, Heterogeneous Multiscale Method: a general methodology for multiscale modeling, Phys. Rev. B: Condens. Matter Mater. Phys., 67, 092101, 2003.

[6] W. E, B. Engquist, X. Li, W. Ren and E. Vanden-Eijnden, Heterogeneous multiscale methods: a review, Commun. Comput. Phys., 2, 367-450, 2007.

[7] W. E, P. Ming and P. Zhang, Analysis of the heterogeneous multiscale method for elliptic homogenization problems, J. Amer. Math. Soc., 18, 121-156, 2004.

[8] B. Engquist, H. Holst and O. Runborg, Multiscale Methods for the Wave Equation, in Sixth International Congress on Industrial Applied Mathematics (ICIAM07) and GAMM Annual Meeting, Wiley, 7, 2007.

[9] B. Engquist and P.E. Souganidis, Asymptotic and numerical homogenization, Acta Numer., $17,147-190,2008$

[10] B. Engquist and Y.H. Tsai, Heterogeneous multiscale methods for stiff ordinary differential equations, Math. Compt., 74, 1707-1742, 2005.

[11] L.C. Evans, Partial Differential Equations, American Mathematical Society, 1998.

[12] V.V. Jikov, S.M. Kozlov and O.A. Oleinik, Homogenization of Differential Operators and Integral Functions, Springer, 1991.

[13] I.G. Kevrekidis, C.W. Gear, J. Hyman, P.G. Kevekidis and O. Runborg, Equation-free, coarsegrained multiscale computation: enabling microscopic simulators to perform system-level tasks, Commun. Math. Sci., 715-762, 2003.

[14] M.G. Krein and M.A. Ruthman, Linear Operators That Leave Invariant a Cone in a Banach Space, Usp. Mat. Nauk., 1948.

[15] X. Li and W. E, Multiscale modelling of the dynamics of solids at finite temperature, J. Mech. Phys. Solids, 53, 1650-1685, 2005.

[16] V.A. Marchenko and E.Y. Khruslov, Homogenization of Partial Differential Equations, Progress in Mathematical Physics, 46, 2006.

[17] P. Ming and X. Yuen, Numerical methods for multiscale elliptic problems, J. Comput. Phys., $214,421-445,2005$.

[18] G. Nguetseng, A general convergence result for a functional related to the theory of homogenization, SIAM J. Math. Anal., 20, 608-623, 1989.

[19] G. Samaey, Patch Dynamics: Macroscopic Simulation of Multiscale Systems, PhD thesis, Katholieke Universiteit Leuven, 2006.

[20] F. Santosa and W.W. Symes, A dispersive effective medium for wave propagation in periodic composites, SIAM J. Appl. Math., 51, 984-1005, 1991. 